\title{
Water Network Optimization with Wastewater Regeneration Models
}

\author{
Linlin Yang $^{\dagger}$, Raquel Salcedo-Diaz ${ }^{\ddagger}$ and Ignacio E. Grossmann ${ }^{\dagger *}$ \\ ${ }^{\dagger}$ Department of Chemical Engineering, Carnegie Mellon University, Pittsburgh, PA \\ ${ }^{\ddagger}$ Department of Chemical Engineering, University of Alicante, Alicante, Spain
}

July, 2014

\begin{abstract}
The conventional water network synthesis approach greatly simplifies wastewater treatment units by using fixed recoveries, creating a gap for their applicability to industrial processes. This work describes a unifying approach combining various technologies capable of removing all the major types of contaminants through the use of more realistic models. The following improvements are made over the typical superstructure-based water network models. First, unit-specific short-cut models are developed in place of the fixed contaminant removal model to describe contaminant mass transfer in wastewater treatment units. Short-cut wastewater treatment cost functions are also incorporated into the model. In addition, uncertainty in mass load of contaminant is considered to account for the range of operating conditions. Furthermore, the superstructure is modified to accommodate realistic potential structures. We present a modified Lagrangean-based decomposition algorithm in order to solve the resulting nonconvex Mixed-integer Nonlinear Programming (MINLP) problem efficiently. Several examples are presented to illustrate the effectiveness and limitations of the algorithm for obtaining the global optimal solutions.
\end{abstract}

\section{Introduction}

With increasing costs, diminishing quality of supplies, and stricter environmental effluent standards set forth by the Environmental Protection Agency (EPA), water is playing an increasingly important role in the process industries. The primary water uses are process water, cooling water, and boiler feed water, with each use being emphasized by different industries. For example, the chemicals, petroleum refining, and metal sectors primarily use water for cooling, while paper and pulp and food processing mostly use water for process use. In a study by Carbon Disclosure Projects of 137 companies with total assets over $\$ 16$ trillion, it has been reported that water has risen high on the corporate agenda[1]. Eighty nine percent of responding companies have developed specific water policies, strategies, and plans. Specifically, in the chemical sector, all ten companies surveyed recognize that there is a high growth potential for processes and products that support more efficient water use and water recycling. Consequently, it is essential to incorporate reuse schemes at the process design level for optimal water use. 
Mathematical programming approaches have been proposed to optimize water networks (WN) using NLP or MINLP models. In a typical water network (WN) superstructure, water is supplied to water-using process units, and then wastewater streams generated from these processes are treated in various treatment units. This versatile superstructure (shown in Figure 1) considers systematic alternatives for water reuse, recycle, and recycle-reuse to minimize freshwater consumption, or more generally, total network cost subject to a specified discharge limit[2]. Variations of this superstructure have been considered in previous works for grassroot designs, namely, considering either only water-using process units[3], focusing only on wastewater treatment units[4], or on both [2, 5-7]. In addition, retrofit of industrial water systems has also been considered[8]. Another approach to WN synthesis is the pinch analysis inspired by heat- and mass-exchange network synthesis. Many studies have been performed to integrate wastewater treatment systems in industrial plants using both approaches $[9,10]$. Thorough reviews of mathematical programming approaches to WN synthesis can be found in [11] and [12], and a review on insight-based methods is presented by Foo[13].

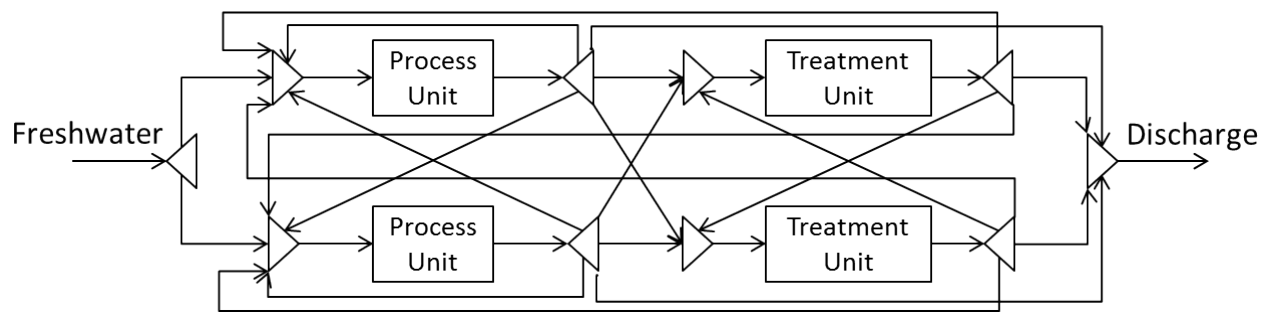

Figure 1: Water network superstructure

The majority of the works related to water network (WN) optimization in the literature assume that the network operates at steady state. Generally speaking, the standard formulation for a WN design problem consists of the following information. The process units in the water network are usually characterized by concentration limits of the entering stream and mass load of contaminants released from the unit, whereas the treatment units are characterized by fixed recoveries (i.e. $C_{j}^{o u t}=\beta_{t j} C_{j}^{i n}$, where $\beta_{t j}$ is the recovery of contaminant $j$ in treatment unit $t ; C_{j}^{i n}$ and $C_{j}^{o u t}$ are contaminant concentration levels at the inlet and the outlet of $t$ ). These models greatly simplify the water network design, but create a gap for their applicability to industrial processes since more accurate treatment models should be considered in the optimization of these water networks.

In order to gain a better understanding of the individual treatment units, it is useful to first consider the treatment procedures of a centralized wastewater treatment plants[14]. In a typical plant, oil and grease are removed in the pretreatment stage. Primary treatment involves the use of physical and chemical operations to remove suspended particles. The next step is secondary treatment, where microorganisms are required to stabilize waste components. Finally, tertiary treatment further removes nitrogen, phosphorus, heavy metals, and bacteria. 
The objective of this work is to gain a more thorough understanding of the trade-offs between the removal efficiency of the treatment units and the cost of the units, as well as their impact on the $\mathrm{WN}$ design. This work combines various technologies capable of removing the three major types of contaminants, namely, total dissolved solids (TDS), total suspended solids (TSS), and organics (ORG), through the use realistic treatment unit models. A number of features are considered in order to achieve this goal and they are described below.

First, unit-specific short-cut models based on the literature are developed to replace the fixed recovery model to more accurately describe contaminant mass transfer in wastewater treatment units. Even though short-cut models have been used in the context of wastewater treatment optimization problem, they usually pertain to specific treatment technologies. For example, Saif et al[15] designed a reverse osmosis network for desalination processes. In contrast, in this work we consider multiple types of treatment units for general processes. To this end, appropriate modeling equations that can satisfactorily predict unit performance with reasonable computational complexity are presented.

Short-cut wastewater treatment cost functions (operating cost and investment cost) in the form of nonlinear functions are incorporated into the model. The conventional network cost function usually consists of a linear operating cost term and a concave capital cost term. The use of a more complex objective in this more rigorous model enables the design of WNs that allow for trade-offs that better meet the need of their respective decision criteria.

In addition, since conditions for a given process may change during the course of the operation, we account for the uncertain parameters through the use of a three-scenario model. This method was demonstrated by Karuppiah and Grossmann[16], where the authors present a multiscenario nonconvex MINLP model that is a deterministic equivalent of a two-stage stochastic programming model with recourse. For each of the best, worst, and nominal scenarios, the uncertain parameters can take on a different set of values. This model then ensures that the final design solution is feasible and optimal over the set of all three scenarios. This representation can effectively capture the wide range of operating conditions without overly complicating the formulation.

Furthermore, the topology of the superstructure is modified to accommodate realistic potential structures. Faria and Bagajewicz[17] explored the impact various topologies among the subsystems has on freshwater consumption of the overall water network. Different types of contaminants present in the system are removed by considering the Best Available Techniques (BAT)[18]. These provide the industrial standards for discharge of the major pollutant groups and recommendations for their treatment as listed in Table 1. Since there are multiple treatment technologies for the removal of each type of pollutants, the modified superstructure (Figure 2) allows for the selection of a subset of BAT treatment technology through the use of disjunctions in the generalized disjunctive programming (GDP) formulation[2].

The resulting multiscenario GDP formulation associated with the WN synthesis problem is computationally ex- 
Table 1: Best available techniques (BAT)

\begin{tabular}{lccccc}
\hline \hline & $\begin{array}{c}\text { Suspended } \\
\text { Solids (TSS) }\end{array}$ & $\begin{array}{c}\text { Heavy Metals } \\
\text { (HM) }\end{array}$ & $\begin{array}{c}\text { Inorganic Salt } \\
\text { (TDS) }\end{array}$ & $\begin{array}{c}\text { Organic Unsuitable } \\
\text { for Bio. Treat (ORG) }\end{array}$ & $\begin{array}{c}\text { Organic Suitable for } \\
\text { Bio. Treat (BOD) }\end{array}$ \\
\hline Sedimentation & $\checkmark$ & $\checkmark$ & & & \\
Flotation & $\checkmark$ & $\checkmark$ & & & \\
Filtration & $\checkmark$ & $\checkmark$ & & & \\
Ultrafiltration & $\checkmark$ & & & & \\
Precipitation & & $\checkmark$ & $\checkmark$ & & \\
$\begin{array}{l}\text { Ion Exchange } \\
\text { Reverse Osmosis }\end{array}$ & $\checkmark$ & $\checkmark$ & $\checkmark$ & & \\
Evaporation & & & & $\checkmark$ & $\checkmark$ \\
$\begin{array}{l}\text { Oxidation } \\
\text { Adsorption } \\
\text { Anaerobic Treatment } \\
\text { Aerobic Treatment }\end{array}$ & & & & $\checkmark$ & \\
\hline
\end{tabular}

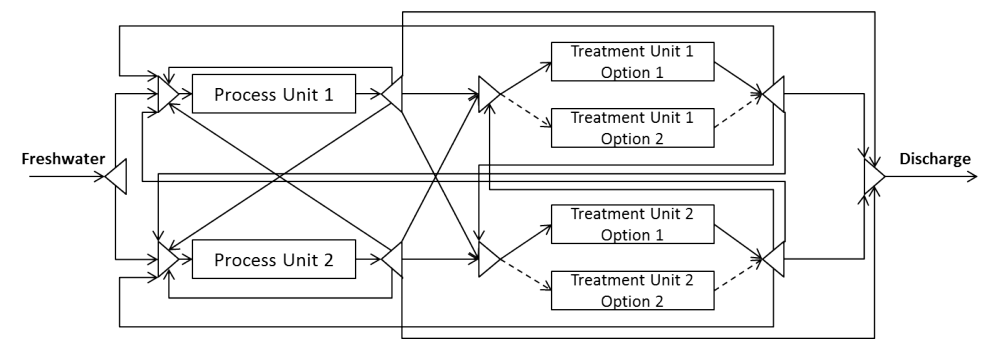

Figure 2: Superstructure with multiple treatment unit options

pensive to solve to global optimality. Various methods have been proposed to address the issue of bilinear terms (products of flowrates and contaminant concentrations) and concave cost functions in the standard water network synthesis problems $[2,19,20]$. The short-cut models presented in this paper introduce additional nonlinear and nonconvex terms. To overcome the difficulty, we first reformulate the GDP problem into a nonconvex MINLP problem. We then present a modified Lagrangean-based decomposition algorithm in order to solve the resulting MINLP problem effectively. The formulation and the effectiveness of the algorithm are then illustrated through applications in metal finishing and petroleum refining industries.

\section{Problem statement}

\subsection{Problem description}

In this manuscript we consider an integrated multi-contaminant $\mathrm{WN}$ with a given set of process units (PU, e.g. scrubber, cooling tower), a set of treatment units ( $T U$, e.g. reverse osmosis, sedimentation), freshwater sources (e.g. lake, municipal treatment plant, water from process separations), and wastewater discharge sinks (e.g. river, 
centralized wastewater treatment plant, cooling tower). These units are interconnected using mixer units ( $M U)$ and splitter units $(S U)$ to form the superstructure, and are shown in Figure 1. Freshwater sources that vary in maximum flowrate and pollutant levels are supplied to one or more of the process units. Once the streams are treated, they are recycled to the process untis or sent to wastewater discharge sinks that must satisfy limits on either the pollutant discharge concentration or on the discharge flowrate.

Each process unit has a fixed water flowrate requirement, upper limits on the inlet concentration level, and mass load of contaminants released into the water stream. The mass load of contaminant is the uncertain parameter that can take a range of values during process operation. We define its upper bound as the worst case scenario, its lower bound as the best case scenario, and the average as the nominal scenario. In comparison to a single steady state scenario design, the proposed model is defined over the three scenarios $n \in N$ that account for the uncertainties in the loads by introducing flexibility to the network design. This network flexibility can be achieved by increasing pipe capacity, piping connections, or treatment unit capacity and removal efficiency.

The standard wastewater treatment units considered in this work include the followings: sedimentation, ultrafiltration, ion exchange, reverse osmosis, activated sludge, and trickling filter. By substituting the simplified models with short-cut models more accurate design can be obtained. The goal is to select a subset of technologies that best fit the treatment applications of the receiving wastewater streams.

\subsection{General model}

The general problem formulation (GDP-1) is an extension of earlier works by Karuppiah and Grossmann and Ahmetović and Grossmann[2, 16, 20]. The main difference here is that the fixed recovery treatment units are replaced by 
short-cut models presented in Section 4. The model (GDP-1) based on the superstructure in Figure (1) is as follows:

$$
\begin{aligned}
\operatorname{min.} \text { Cost }^{\text {total }} & =A R \sum_{t \in T U} I C_{t}^{T U}+A R\left[\sum_{i \in \text { Pipe }}\left(C_{i}^{\text {Pipe }} y_{i}+I C_{i}^{\text {Pipe }}\left(\hat{F}_{i}\right)^{\delta}\right]\right. \\
+ & H \sum_{w} \sum_{n} p_{n} O C^{F W} F W_{n}^{w}+H \sum_{n} p_{n} \sum_{i \in \text { Pipe }} O C^{\text {Pipe }} F_{i n}+\sum_{n} p_{n} O C_{t n}^{T U}
\end{aligned}
$$

s.t. $\quad F_{k n}=\sum_{i \in m_{\text {in }}} F_{\text {in }} \quad \forall m \in M U, k \in m_{\text {out }}, \forall n \in N$

$$
\begin{aligned}
& F_{k n} C_{k j n}=\sum_{i \in m_{\text {in }}} F_{i n} C_{i j n} \quad \forall j, \forall m \in M U, k \in m_{\text {out }}, \forall n \in N \\
& F_{k n}=\sum_{i \in s_{\text {out }}} F_{\text {in }} \quad \forall s \in S U, k \in s_{\text {in }}, \forall n \in N \\
& C_{i j n}=C_{k j n} \quad \forall j, \forall s \in S U, i \in s_{\text {out }}, k \in s_{\text {in }}, \forall n \in N \\
& F_{k n}=F_{\text {in }}=P_{p}^{P U} \quad \forall p \in P U, i \in p_{\text {in }}, k \in p_{\text {out }}, \forall n \in N \\
& P_{p}^{P U} C_{k j n}+L_{p j n} \times 10^{3}=P_{p}^{P U} C_{i j n} \quad \forall j, \forall p \in P U, k \in p_{\text {in }}, i \in p_{\text {out }}, \forall n \in N \\
& \mathrm{r}=1, \ldots, R T_{t}\left[\begin{array}{c}
Y_{r t} \\
h_{n}\left(d_{r t}, F_{\text {in }}, C_{i j n}\right)=0 \\
g_{n}\left(d_{r t}, F_{\text {in }}, C_{i j n}\right) \leq 0 \\
I C_{t}^{T U}=f_{1}\left(d_{r t}\right) \\
O C_{t n}^{T U}=f_{2}\left(d_{r t}, F_{i n}, C_{i j n}\right)
\end{array}\right] \forall j, \forall t \in T U, i \in t_{\text {in }} \cup t_{\text {out }}, \forall n \in N
\end{aligned}
$$

$$
Y_{r t} \in\{\text { True, False }\}
$$

$$
\hat{F}_{i} \geq F_{\text {in }} \quad \forall i, \forall n \in N
$$$$
y_{i} \in[0,1] \quad \forall i
$$$$
F_{i}^{M I N} y_{i} \leq \hat{F}_{i} \leq F_{i}^{M A X} y_{i} \quad \forall i
$$$$
F_{i}^{M I N} \leq F_{i n} \leq F_{i}^{M A X} \quad \forall i, \forall n \in N
$$$$
C_{i j}^{M I N} \leq C_{i j n} \leq C_{i j}^{M A X} \quad \forall j, \forall i, \forall n \in N
$$

(GDP-1)

where $y_{i}$ are binary variables to indicate existence of piping connection $i ; F_{i n}$ and $F_{k n}$ are flowrates (t/h) of any stream $i$ and $k$ in the superstructure respectively, in scenario $n ; \hat{F}_{i}$ is the maximum flowrate capacity of pipe $i, C_{i j n}$ and $C_{k j n}$ are concentrations ( $\mathrm{ppm}$ ) of contaminant $j, P_{p}^{P U}$ are the process unit water flowrates, $L_{p j n}$ are the mass load of contaminant $j$ in unit $p$ in scenario $n(\mathrm{~kg} / \mathrm{h})$. In the disjunctive formulation, $Y_{r t}$ indicates if technology $r$ is chosen for unit $t, d_{r t}$ is the design variable associated with $r$ and $t$. The constraints consist of a set of contaminant mass balances in the mixer units, splitter units, process units, and treatment units $\left(h_{n}(\bullet), g_{n}(\bullet)\right)$. Note that for the set of splitters $S U$, there is a subset of initial splitters $S U^{w}$ for which $F_{k n}^{w}=F W_{n}^{w}, w \in W$, where $W$ is set of freshwater sources. 


\subsection{Objective function}

The objective function of the problem is to minimize the total cost of the network $\left(\right.$ Cost $\left.^{\text {total }}\right)$. It consists of the annualized investment cost and the expected operating cost. The investment cost is scenario independent and is given by the sum of treatment unit capital costs $\left(I C_{t}^{T U}\right)$ and pipe investment costs (the second term in the objective function). $C_{i}^{\text {Pipe }}$ are the fixed charge cost coefficients (\$) associated with pipe existence, and $I C_{i}^{\text {Pipe }}$ are the investment cost coefficients of pipes, and $\delta$ is the associated cost exponent. The expected operating cost of the network represents the operating cost for the selected a network design over all three scenarios, each with a given probability $p_{n}$. The term includes freshwater cost, pumping cost, and treatment unit operating cost. $O C^{F W, w}$ are the cost coefficients of freshwater sources $(\$ / \mathrm{t}), O C^{\text {Pipe }}$ is the pumping cost coefficient $(\$ / \mathrm{t})$, and $O C_{t n}^{T U}$ is the treatment unit operating cost. $H$ is the operating hours in a year (hr/year), and $A R$ is the annualized factor for investment cost (year ${ }^{-1}$ ).

Treatment unit cost equations are greatly simplified in previous works on WN optimization. Specifically, the treatment unit capital costs are usually modeled as a concave function of the inlet flow, and the operating cost as a linear function of the inlet flowrate as shown in Equation 1.

$$
\begin{aligned}
I C_{t} & =C I C_{t}\left(F_{i}\right)^{\alpha} \\
O C_{t} & =C O C_{t} F_{i}
\end{aligned}
$$

where $C I C_{t}$ and $C O C_{t}$ are cost coefficients for investment cost and operating cost, respectively. In this work, we incorporate treatment unit cost correlations that are function of design variables such as area or volume of the unit to reduce the gap between the true total cost of the network and the objective obtained from the simplified optimization model.

\section{Illustrative example}

In order to demonstrate the advantage of performing multi-scenario optimization, we present an illustrative example with two process unit/two sets of treatment units (two options each)/two contaminants system with data given in Table 2. We solve the example using the worst case scenario model (i) and the three-scenario model (iii). The worst case scenario model optimizes over scenario (n1) only. To obtain an accurate comparison between the two solutions, we solve an additional three-scenario model (ii) subject to piping connectivity and flowrate capacity bound obtained from the worst case scenario model (i).

The resulting network costs are presented in Table 3, where it can be seen that the worst case design (i) operating in the 3 scenarios (ii) is $\$ 22,820$ more expensive than the design that was optimized for the 3 scenarios (iii). As shown 
Table 2: Illustrative example data

(a) Process units

(b) Treatment units

\begin{tabular}{|c|c|c|c|c|c|c|c|c|c|c|c|c|c|c|c|c|c|c|c|}
\hline & & ptions & \multicolumn{6}{|c|}{ Removal Ratio (\%) } & \multirow[t]{3}{*}{$\mathrm{IC}(\$)$} & \multirow[t]{3}{*}{$\mathrm{CO}(\$ /$ ton $)$} \\
\hline & \multirow[t]{3}{*}{ Flowrate (ton/h) } & \multicolumn{6}{|c|}{ Discharge Load $(\mathrm{kg} / \mathrm{h})$} & \multirow{2}{*}{\multicolumn{2}{|c|}{$C_{i n}^{\max }(\mathrm{ppm})$}} & & & \multicolumn{2}{|c|}{$\mathrm{n} 1$} & \multicolumn{2}{|c|}{$\mathrm{n} 2$} & \multicolumn{2}{|c|}{ n3 } & & \\
\hline & & $\mathrm{n}$ & & $\mathrm{n}$ & & n? & & & & & & A & $\mathrm{B}$ & A & B & A & $\mathrm{B}$ & & \\
\hline & & $\mathrm{A}$ & $\mathrm{B}$ & A & $\mathrm{B}$ & $\mathrm{A}$ & $\mathrm{B}$ & A & B & & OP1 & 90 & 0 & 95 & 0 & 99 & 0 & 16800 & 1 \\
\hline PU1 & 40 & 1.1 & 1.7 & 1 & 1.5 & 0.8 & 1.3 & 0 & 0 & TU1 & OP2 & 80 & 0 & 90 & 0 & 98 & 0 & 4800 & 0.5 \\
\hline PU2 & 50 & 2 & 2 & 1.7 & 1.8 & 1.5 & 1.6 & 50 & 50 & & OP1 & 0 & 80 & 0 & 90 & 0 & 95 & 12600 & 0.0067 \\
\hline & & & & & & & & & & 102 & OP2 & 0 & 90 & 0 & 95 & 0 & 99 & 36000 & 0.067 \\
\hline
\end{tabular}

in Figure 3, both cases (i) and (iii) select Option 2 for TU1 and Option 1 for TU2. The difference lies in the number of piping connections - 8 removable pipes in the superstructure are determined by model (i) vs 12 removable pipes in model (iii). A removable pipe is a piping connection between a mixer unit and a splitter unit. As a result, case (iii) allows for additional flexibility. Specifically, it allows for the bypass stream (PU1, discharge mixer unit) in the best scenario (n3). The bypass stream is not selected in the worst-scenario model. Thus, the flow is redirected to PU2 and treatment units, increasing the treatment cost.

Table 3: Illustrative example optimization results

\begin{tabular}{lrrrr}
\hline \hline & (i) Worst case & (ii) Comparison with three-scenario & (iii) Three-scenario \\
\hline \# of removable pipes & 8 & 8 & 12 \\
Annualized IC $(\$ / y r)$ & $39,426.50$ & $39,430.60$ & $39,821.43$ \\
Operating cost $(\$ / y r)$ & $634,742.00$ & $526,398.20$ & $503,187.22$ \\
Total cost $(\$ / y r)$ & $674,161.40$ & $565,828.80$ & $543,008.65$ \\
\hline
\end{tabular}

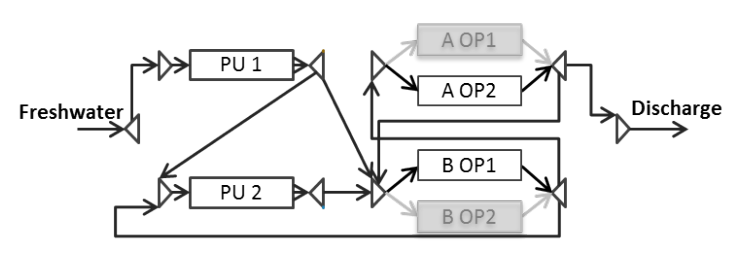

(a)

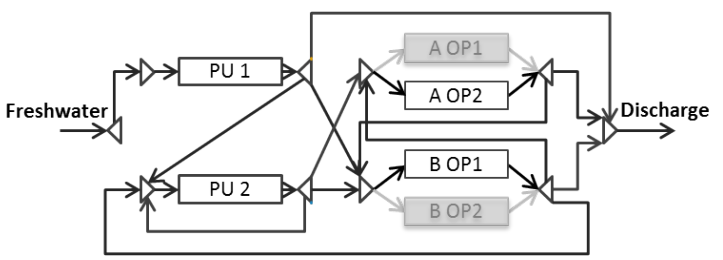

(b)

Figure 3: Illustrative example result: (a) Worst case scenario (b) Three-scenario

The example was solved with BARON[21] and the computational statistics are presented in Table 4. The large CPU time required in the three scenario case clearly indicates that a suitable decomposition scheme is required for these problems. 
Table 4: Computational statistics for illustrative example

\begin{tabular}{lrr}
\hline \hline & (i) Worst case & (iii) Three-scenario \\
\hline \# of constraints & 229 & 575 \\
\# of continuous vars & 161 & 431 \\
\# of integer vars & 24 & 24 \\
CPU time (s) & 25 & $1800^{*}$ \\
Optimality gap (\%) & 4.98 & 15.6 \\
\hline${ }^{*}$ Time limit
\end{tabular}

\section{Wastewater treatment unit short-cut models}

The purpose of this section is to describe a set of common treatment units mentioned previously, and to consider their performance as well as important design considerations. Treatment unit models with various levels of detail have been reported in literature. The models reported here aim to describe each unit adequately while minimizing computational complexity. To the knowledge of the authors these models have not been incorporated into WN superstructure optimization. A list that summarizes the unit-specific variable names is presented in the nomenclature section.

For the sake of clarity, in this section we denote treatment unit inlet flowrate by $Q_{0}\left(\mathrm{~m}^{3} /\right.$ day), outlet flowrate by $Q$ $\left(\mathrm{m}^{3} /\right.$ day), inlet contaminant concentration by $S_{0}^{j}(\mathrm{ppm})$, treated outlet contaminant concentration by $S^{j}(\mathrm{ppm})$, contaminant $j$ removal ratio by $R c^{j}$, and flow recovery ratio by $R r$. The recovery $R r$ is assumed to be 1 for sedimentation, ion exchange, and trickling filter. They are related as follows.,

$$
\begin{aligned}
Q & =R r Q_{0} \\
S^{j} & =\left(1-R c^{j}\right) S_{0}^{j}
\end{aligned}
$$

\subsection{Reverse osmosis}

Reverse osmosis (RO) is a pressure-driven membrane treatment process mainly used in seawater and brackish desalination applications. A high-pressure feed stream flows across the surface of a semi-permeable material. Due to a pressure differential between the feed and permeate sides of the membrane, a portion of the feed stream passes through the membrane. The permeate stream exits at nearly atmospheric pressure, while the concentrate remains at nearly the feed pressure. The salt rejection coefficient $\left(R c^{T D S}\right)$ limits the membrane performance and its value is fixed for a specific membrane. The value of the recovery ratio $(R r)$ usually lies between 0.5 and 0.9 . A scheme of the RO process is shown in Figure 4.

The performance of the system depends mainly on two parameters in the RO process design, they are the transmembrane pressure $\Delta P$ and the membrane area $A_{m e m b}$. The selected type of membrane element is the spiral bound FILMTEC BW30-400 (DOW) that offers high flow and rejection. The membrane properties are specified by the 


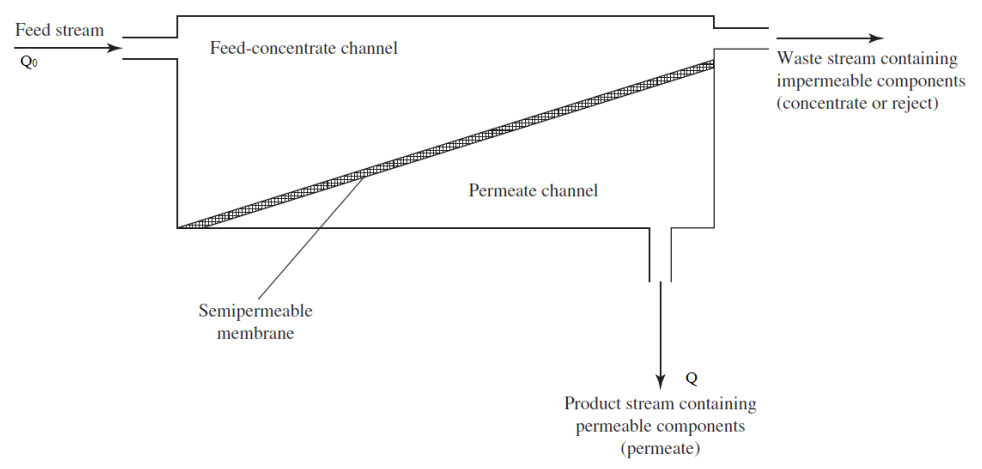

Figure 4: Reverse osmosis diagram[14]

manufacturer and they are shown in Table 5.

Mass transfer in RO involves a diffusive mechanism such that separation efficiency is dependent on influent solute concentration, pressure, and water flowrate. The permeate flowrate across the membrane is determined by the osmotic pressure law (3),

$$
Q=A_{m e m b} N k_{m}(\Delta P-\Delta \pi)
$$

The transmembrane pressure $(\Delta P)$ is calculated as in (4)[22],

$$
\Delta P=P_{f}-P_{p}-\frac{\Delta P_{\text {drop }}}{2}
$$

where $P_{f}$ is the feed stream pressure, $P_{p}$ is the permeate stream pressure.

Assuming the feed stream is a dilute solution of salts, the osmotic pressure $\pi$ can be approximated by the Van't Hoff equation in (5),

$$
\Delta \pi=\frac{\phi R T}{M}\left(S_{0}^{T D S}-S^{T D S}\right)
$$

It is also assumed that the concentration polarization is negligible so that the concentration at the membrane surface is considered to the be equal to the concentration at the inlet of the RO treatment $\left(\mathrm{C}_{\mathrm{f}}\right)$.

\subsection{Ion exchange}

Ion exchange (IX) is a reversible reaction in which a charged ion in solution is exchanged for a similarly charged ion electrostatically attached to an immobile solid particle. In practice the raw water is commonly passed through a bed of resin. When the bed becomes saturated with the exchanged ion, it is shut down and regenerated by passing 


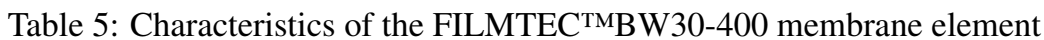

\begin{tabular}{lclr}
\hline \hline Parameter & Symbol & Unit & Value \\
\hline Membrane rejection coefficient & $\mathrm{Rc}$ & & 0.98 \\
Membrane water permeability & $k_{m}$ & $\mathrm{t} /\left(\right.$ day m $\left.{ }^{2} \mathrm{~Pa}\right)$ & $6.48 \times 10^{-7}$ \\
Membrane area & $\mathrm{A}$ & $\mathrm{m}^{2}$ & 37 \\
Gas constant & $\mathrm{R}$ & $\mathrm{kJ} /(\mathrm{kmol} \mathrm{K})$ & 8.31 \\
Max pressure drop in vessel & $\Delta P_{\text {drop }}$ & $\mathrm{bar}$ & 3.4 \\
Number of ions in solution & $\phi$ & & 2 \\
Molar mass of the dissolved solids & $\mathrm{M}$ & $\mathrm{g} / \mathrm{mol}$ & 58.44 \\
\hline
\end{tabular}

a concentrated solution of the presaturant ion back through the bed. The saturation of the resin is shown in the breakthrough curve (Figure 5a). At the break point, the effluent concentration exceeds the design criteria and the column needs to be regenerated. Figure $5 b$ shows a scheme of a typical IX column configuration.

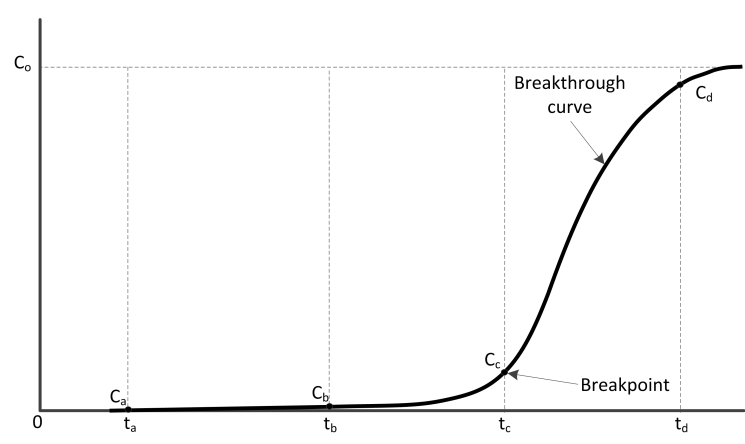

(a)

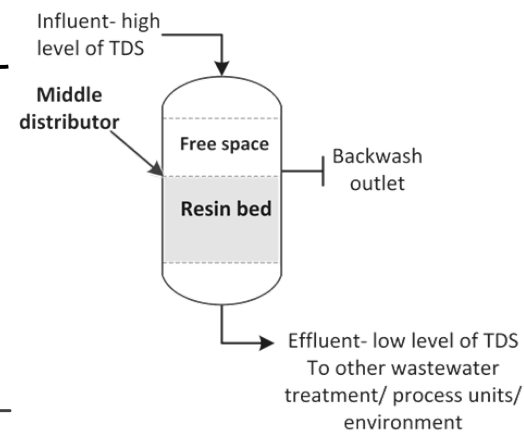

(b)

Figure 5: Ion exchange unit diagrams: (a) Ion exchange breakthrough curve, (b) Ion exchange column configuration diagram: (i) Loading cycle (ii) Regeneration cycle[23]

For the complete removal of ions the water stream must pass through cationic and anionic resins in series or through a unique column containing a mixture of both. The performance of the system depends on many parameters such as the operating capacity $(q)$, the service flow rate $(S F R)$ or the surface loading rate $(S L R)$, which determines the pressure drop in the resin. $B V$ is the volume of water treated per volume of resin, and it relates the concentration gradient with the capacity of the resin bed,

$$
B V=1000 \frac{q}{S^{T D S}-S_{0}^{T D S}}\left(X M W_{c a}+(1-X) M W_{a n}\right)
$$

where $X$ is the mass fraction in inlet water of ion wanted to be removed, $M W_{c a}$ is the molar mass of the cation, and $M W_{a n}$ is the molar mass of the anion.

SFR is determined from the following equation, and the typical SFR ranges from 8 to 40 bed volume per hour 
$(\mathrm{BV} / \mathrm{h})$.

$$
S F R=\frac{B V}{C T}
$$

where $C T$ is the contact time.

The process design variables can be modeled with the equations in (8),

$$
\begin{aligned}
V & =\frac{Q_{0}}{S F R} \\
V_{w w} & =\frac{q}{S_{0}-S} \\
A & =\frac{Q_{0}}{S L R}
\end{aligned}
$$

where $V$ is the resin volume, $V_{w w}$ is the volume of wastewater treated, and $A$ is the resin cross-sectional area.

Some design considerations for determining model parameters are as follows[14]. The pressure drop in the bed should be kept in the range of 35-70 kPa, with a maximum value of $135 \mathrm{kPa}$. This results in a maximum $S L R$ of 880 $\mathrm{m} /$ day, depending on the resin. Regarding the operating parameters, $S F R$ should be kept in the range of 8 to $40 \mathrm{BV} / \mathrm{h}$ to ensure an adequate contact time and to avoid an early breakthrough.

\subsection{Sedimentation}

Sedimentation is used as a preliminary step to reduce TSS level in wastewater streams. Typically, 50 to $70 \%$ of TSS and 25 to $40 \%$ of BOD can be removed using primary sedimentation tanks [14]. The standard sedimentation tanks are of circular or rectangular design, whose selection is determined by a number of factors. Figure 6 is a schematic drawing of a horizontal flow tank.

The efficiency of sedimentation tanks is affected by a number of factors including eddy currents formed by the inertia of the incoming fluid, thermal convection currents, and density currents caused by cold or warm water along the bottom of the tank and warm water flowing across the top of the tank.

Typical removal performance $\left(R c^{j}\right)$ of a rectangular tank can be modeled by a hyperbolic function (9) of the detention time $(t)$ and contaminant $(j)[24]$.

$$
R c^{j}=1-\frac{t}{a_{j}+b_{j} t}
$$




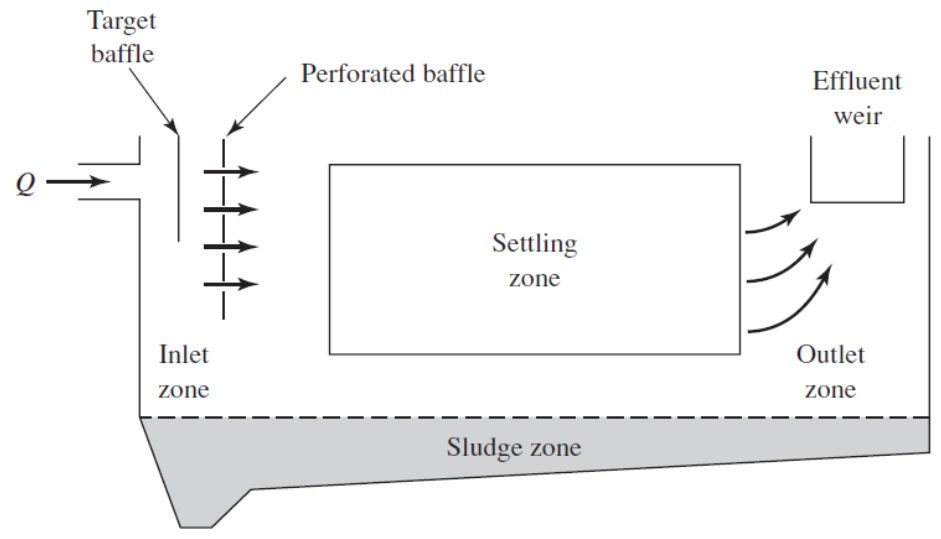

Figure 6: Horizontal flow sedimentation diagram[14]

where $a, b$ are empirical constants presented in Table 6 .

Table 6: Typical values for the empirical constants at $20^{\circ} \mathrm{C}$

\begin{tabular}{lcc}
\hline Contaminant & $\mathrm{a}$ & $\mathrm{b}$ \\
\hline BOD & 0.018 & 0.020 \\
TSS & 0.0075 & 0.014 \\
\hline
\end{tabular}

The design of the rectangular tank can be calculated by the following equations,

$$
\begin{aligned}
A & =Q / O R \\
D & =t Q / A \\
N C & =\frac{A}{L W}
\end{aligned}
$$

where $O R$ is the overflow rate, $A$ is the area, $D$ is the depth, and $N C$ is the number of clarifiers required.

\subsection{Ultrafiltration}

Ultrafiltration (UF) is a pressure driven membrane filtration process. The feed stream is a suspension, or two-phase system, in which the dispersed solid phase to be separated may include sediment, algae, bacteria, protozoa, viruses, or colloids as shown in Figure 7 in which comparisons are presented with microfiltration (MF), nanofiltration (NF), and reverse osmosis (RO). The primary goal of membrane filtration is to produce a product stream (water) from which the targeted solids have been almost completely removed. The predominant removal mechanism in UF is size exclusion so the process can theoretically achieve perfect exclusion of particles regardless of operational parameters such as influent concentration and pressure. UF membranes cover a wide range of molecular weight cutoffs (MWCOs) and 
Table 7: Characteristics of a typical UF membrane

\begin{tabular}{lclr}
\hline \hline Parameter & Symbol & Unit & Value \\
\hline Membrane water permeability & $k_{m}$ & $\mathrm{t} /\left(\right.$ day $\left.\mathrm{m}^{2} \mathrm{~Pa}\right)$ & $1.3704 \times 10^{-5}$ \\
Active area of membrane element & $\mathrm{A}$ & $\mathrm{m}^{2}$ & 33 \\
Max pressure drop in vessel & $\Delta \mathrm{P}$ & bar & $0.4-1.5$ \\
\hline
\end{tabular}

pore sizes. Operational pressures range from 70 to $700 \mathrm{kPa}$, depending on the application [14].

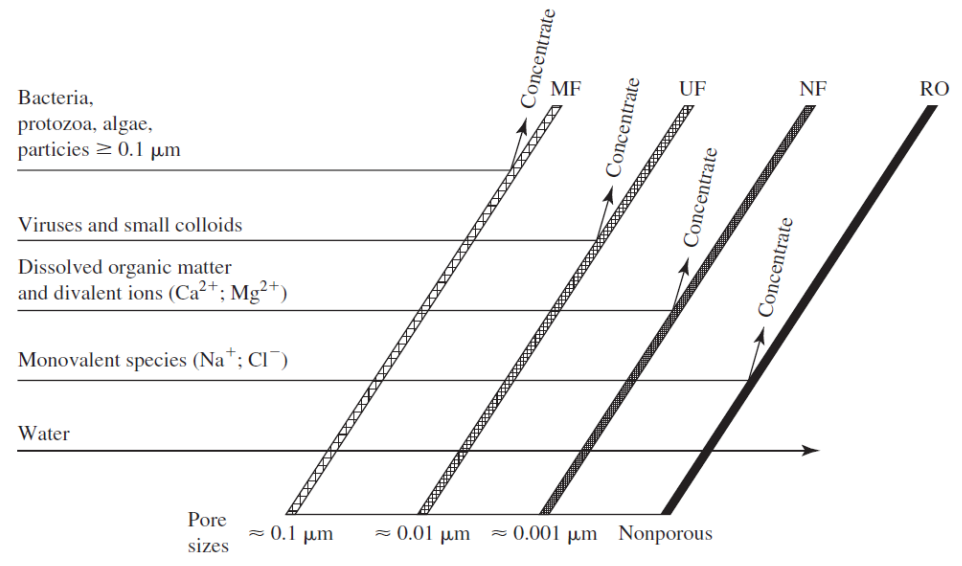

Figure 7: Membrane processes comparison[14]

As shown in Figure 7, the UF process shares some common features with the RO process. The material balances must be satisfied and the feed and permeate concentration are related through the rejection coefficient (2). The recovery ratio is assumed to lie between 0.5 and 0.9 . Pure water transport across a clean porous membrane is directly proportional to the transmembrane pressure $(\Delta P)$. The number of units $(N)$ required is based on the permeate flowrate $(Q)$ as shown in equation (11),

$$
Q=N \Delta P k_{M} A_{m e m b}
$$

Fouling of the membrane may occur during the filtration, which implies an additional resistance to the water flux through the membrane. For the sake of simplicity, we do not consider membrane fouling in the mathematical model. Typical UF membrane characteristics [23] are shown in Table 7.

\subsection{Activated sludge}

Activated sludge (AS) is an aerobic slurry commonly used in wastewater treatment for the removal of soluble organic matters. Microorganisms in the wastewater convert organic matter to biomass and other components in the complete- 


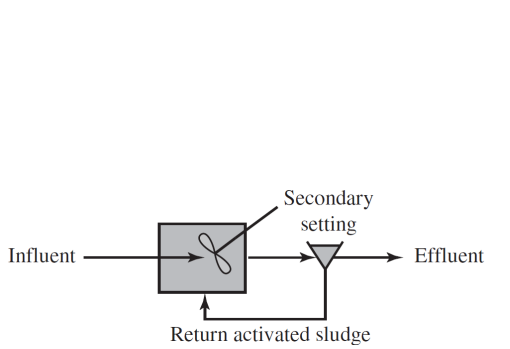

(a)

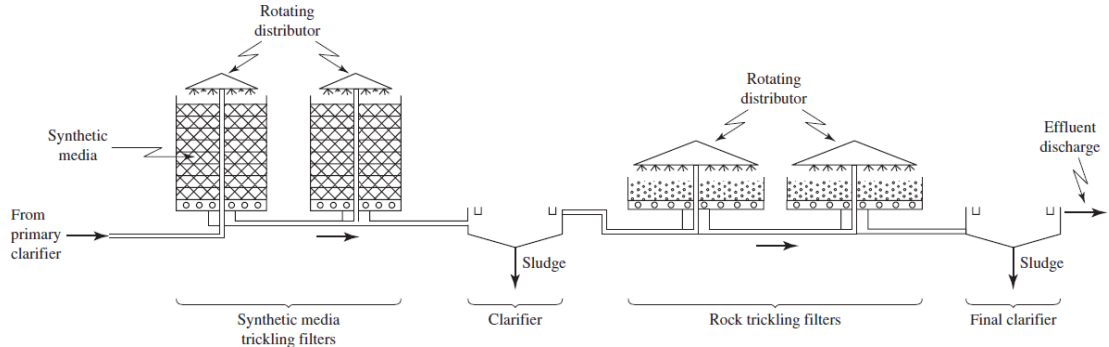

(b)

Figure 8: Organics removal units schematics [14], (a) Activated sludge, (b) Two-stage trickling filter[14]

mix suspended growth reactor. Once the stream exits the reactor, the suspended solids (sludge) are partially removed in a clarifier, while the rest is recycled to the reactor. The removed sludge then goes through a series of sludge treatment to be discharged to the environment. This process is shown in Figure 8a.

An important parameter that determines the system performance is the solid retention time $(S R T)$, which is used to characterize the average time the activated-sludge solids remain in the system [24]. The effluent soluble substrate concentrations in (12), $S$, is only a function of the $S R T$ and kinetic coefficients, and is neither a function of the influent soluble substrate concentration nor the sizes of the reactor. However, the size of the reactor needs to scale with $S R T$ in order to avoid system upsets.

$$
S=\frac{K_{s}\left[1+\left(k_{d}\right) S R T\right]}{S R T\left(Y k-k_{d}\right)-1}
$$

where $K_{s}, k_{d}, Y, k$, and $f_{d}$ are kinetic parameters and their values are given in Table 8 . The process can be modeled with the equations in (13),

$$
\begin{aligned}
X & =\left(\frac{S R T}{\tau}\right)\left[\frac{Y\left(S_{0}-S\right)}{1+\left(k_{d}\right) S R T}\right] \\
X_{T} & =\left(\frac{S R T}{\tau}\right)\left[\frac{Y\left(S_{0}-S\right)}{1+\left(k_{d}\right) S R T}\right]+\left(f_{d}\right)\left(k_{d}\right) X S R T+\frac{\left(X_{0, i}\right) S R T}{\tau} \\
P_{X_{T}, V S S} & =\frac{X_{T} V}{S R T} \\
P_{X, b i o} & =P_{X_{T}, V S S}-Q X_{0, i}
\end{aligned}
$$

where $X$ is the biomass concentration in the aeration tank, $X_{T}$ is MLVSS concentration, $X_{0, i}$ is the influent nbVSS concentration, $P_{x, b i o}$ is biomass wasted, and $P_{X_{T}, V S S}$ is total sludge wasted daily.

In general, the operating cost of the AS is higher than the cost of other secondary treatment processes primarily because of the need to supply molecular oxygen using mechanical aerator, which can be energy-intensive. The oxygen 
Table 8: AS kinetic parameters

\begin{tabular}{llr}
\hline \hline Symbol & Unit & Value \\
\hline$K_{s}$ & g COD/m & 10 \\
$k_{d}$ & g VSS/(g VSS day) & 0.1 \\
$Y$ & g VSS/g COD & 0.4 \\
$k$ & g VSS/(g VSS day) & 12.5 \\
$f_{d}$ & g VSS/g VSS & 0.15 \\
\hline
\end{tabular}

consumption $\left(R_{o}\right)$ is given by the following correlation:

$$
R_{o}=Q\left(S_{0}-S\right)-1.42 P_{x, b i o}
$$

The sizing of the unit is modeled as follows,

$$
\begin{gathered}
V=\tau Q \\
A c l=\frac{Q X_{T}}{S L R}
\end{gathered}
$$

where $V$ is the reactor volume, $\tau$ is the resident time, and $A c l$ is the clarifier area.

\subsection{Trickling filter}

Trickling filter (TF) is a circular packed bed of media covered with a biological film of microorganisms, which operates using attached-growth process. Liquid wastewater is distributed over the top of the unit by a rotary distributor. Oxygen diffuses into the media, and treatment of the wastewater stream is accomplished by the biofilm in the filter. Organic removal rate is related to the available surface area and contact time of the wastewater with the surface [14].

A two-stage trickling filter system is the most typical process used that improves the performance of the unit. The second stage provides additional contact between the organics and the microorganisms on the filter media. The two stages could have different media as shown in Figure 8b. The organic removal ratio $\left(R c^{O R G}\right)$ can be related to the removal efficiency of stage one $\left(E_{1}\right)$ and stage two $\left(E_{2}\right)$ as follows,

$$
R c^{O R G} \times 100=E_{1}+E_{2}\left(1-\frac{E_{1}}{100}\right)
$$

The empirical design equations for BOD removal were developed for rock trickling filters based on the performance at 34 plants at military installations treating domestic wastewater [23]. For a single-stage filter or the first stage of a 
two-stage rock filter, the efficiency at $20^{\circ} \mathrm{C}$ is,

$$
\begin{aligned}
E_{1} & =\frac{100}{\left(1+0.4432 \sqrt{\frac{W_{1}}{V F}}\right)} \\
W_{1} & =Q S_{0} \frac{1 k g}{1000 g}
\end{aligned}
$$

where $W_{1}$ is BOD loading applied to the first-stage filter. For the purpose of this work, we ignore the effect of wastewater temperature on the BOD removal efficiency.

The recirculation factor $F$ represents the average number of passes of the raw wastewater BOD through the filter. The 0.1 factor accounts for the empirical correlation of the decreasing biodegradability with increasing number of passes([14]). The recycle ratio $R_{r c y}$ is typically between 0 and 2 .

$$
F=\frac{1+R_{r c y}}{\left(1+0.1 R_{r c y}\right)^{2}}
$$

The second stage efficiency is given as follows:

$$
\begin{aligned}
E_{2} & =\frac{100}{\left(1+\frac{0.4432}{1-E 1 / 100} \sqrt{\frac{W_{2}}{V F}}\right)} \\
W_{2} & =\left(1-E_{1}\right) W_{1}
\end{aligned}
$$

where $W_{2}$ is BOD loading applied to the second-stage filter.

As with all aerobic treatment operations, an adequate supply of air is crucial to provide efficient treatment of the wastewater stream. The formulation (20) has been developed by Dow Chemical to estimate oxygen consumption $\left(R_{o}\right)$ for trickling filter applications[24],

$$
\begin{aligned}
& R_{o}=20\left(0.8 e^{-9 L_{B}}+1.2 e^{-0.17 L_{B}}\right) \\
& L_{B}=Q S_{0} / V
\end{aligned}
$$

where $L_{B}$ is BOD loading to filter.

Important design variables include the volume of the filter media and the area of the clarifier. The depth, $D$, of 
each filter is typically within $3-11.4 \mathrm{~m}$. Other design parameters include,

$$
\begin{aligned}
O R & =-0.0556 D^{2}+0.7056 D-0.7889 \\
A & =Q / O R \\
V & =A D \\
A c l & =Q / 19.92
\end{aligned}
$$

where $O R$ is overflow rate, $A c l$ is the clarifier area, and $A, D, V$ are area, depth, and volume of the filter media, respectively.

\subsection{Economics of treatment units}

The cost correlations for standard wastewater treatment units can be found in several sources[25, 26]. In addition, we have derived cost equations as functions of unit sizes using the software Superpro Designer [27] through curve fitting. Superpro features end-of-pipe treatment process units for pollution prevention studies.

The investment cost terms $I C_{t}$ are functions of equipment sizing such as area and volume, whereas operating cost $O C_{t}$ include unit throughput, electricity $\left(c_{e}, \$ 0.0981 / \mathrm{kWh}\right)$, material replacement, oxygen consumption $\left(c_{O 2}, \$ 0.02 / \mathrm{kg}\right)$ and waste disposal cost $\left(c_{d}, \$ 0.0001 / \mathrm{kg}\right) . N_{t}$ represent the number of units required to achieve the separation. The equations for the various units are presented below, and their corresponding cost coefficients, $c^{t}$, and cost exponents, $\alpha^{t}$, for the various units are collectively presented in Table $9 . H$ is the number of working hours in a year.

Table 9: Typical cost correlation values

\begin{tabular}{lllllllll}
\hline \hline & $c_{1}$ & $c_{2}$ & $c_{3}$ & $c_{4}$ & $c_{5}$ & $c_{6}$ & $\alpha_{1}$ & $\alpha_{2}$ \\
\hline Reverse Osmosis & 121.35 & 7802.6 & 830 & & & & & \\
Ion Exchange & 8400.7 & $1 \mathrm{e}-13$ & $-2 \mathrm{e}-7$ & 0.1517 & 39162 & & 0.3474 \\
Sedimentation & 8483.8 & 1.69 & 11376 & & & & 0.6 & \\
Ultrafiltration & 138.9 & 303.47 & 400 & & & & \\
Activated Sludge & 241.17 & 8485.9 & 4.58 & 36295 & 3.32 & 5842 & 0.6416 & 0.6 \\
Trickling Filter & -2.4234 & 1731.6 & 69391 & 8485.9 & 3.3445 & 43678 & 0.6 & \\
\hline
\end{tabular}

Reverse osmosis The capital cost is a function of the membrane area, $A_{R O}$, and the operating cost consists of membrane replacement cost, pumping electricity cost, and disposal cost. $L T_{m}$ is the membrane element lifetime (5 years), $\eta_{p}$ is the pump efficiency (0.8), and $\rho^{R O}$ is the feed density $\left(1000 \mathrm{~kg} / \mathrm{m}^{3}\right)$. The operating cost of the RO 
unit is high due to the energy consumption of the high pressure pump.

$$
\begin{aligned}
I C_{R O} & =\left(c_{1}^{R O} A_{R O}+c_{2}^{R O}\right) N_{R O} \\
O C_{R O} & =\frac{c_{3}^{R O} N_{R O}}{L T_{m}}+\frac{c_{e} H \Delta P F_{i n}}{\eta_{p} \rho^{R O}}+c_{d}^{T D S} H F w^{R O}
\end{aligned}
$$

Ion exchange The capital cost is a function of the resin volume, $V_{I} X$, needed for the treatment. The operating cost includes the regenerating cycle (chemicals, brine disposal), which is a polynomial function of the throughput.

$$
\begin{aligned}
& I C_{I X}=c_{1}^{I X}\left(V_{I X}\right)^{\alpha_{1}^{I X}} \\
& O C_{I X}=c_{2}^{I X}\left(F_{i n}\right)^{3}-c_{3}^{I X} F_{i n}^{2}+c_{4}^{I X} F_{i n}+c_{5}^{I X}
\end{aligned}
$$

Sedimentation The capital cost is a function of the area, $A_{S E}$, and the operating cost is a function of throughput and the number of rectangular clarifiers $N C_{S E}$.

$$
\begin{aligned}
I C_{S E} & =c_{1}^{S E} A_{S E}^{\alpha_{1}^{S E}} \\
O C_{S E} & =N C_{S E}\left(\frac{c_{2}^{S E} F_{i n}}{O R_{S E}}+c_{3}^{S E}\right)
\end{aligned}
$$

Ultrafiltration The capital cost mainly depends on the membrane area, $A_{U F}$, needed for the separation. The operating cost include membrane replacement cost, electricity cost, and disposal cost. Since the transmembrane pressure in UF is not so high as in RO, the operational cost is less dependent of the electricity consumption.

$$
\begin{aligned}
I C_{U F} & =\left(c_{1}^{U F} A_{U F}+c_{2}^{U F}\right) N_{U F} \\
O C_{U F} & =\frac{c_{3}^{U F} N_{U F}}{L T m}+\frac{c_{e} H \Delta P F_{i n}}{\eta_{p} \rho_{U F}}+c_{d}^{T S S} H F w_{U F}
\end{aligned}
$$

Activated sludge The capital cost is a function of aeration basin volume $V_{A S}$ and clarifier area $A c l_{A S}$. The operating cost has three terms: maintenance cost as a function of throughput, oxygen consumption, and sludge disposal cost.

$$
\begin{aligned}
I C_{A S} & =c_{1}^{A S}\left(V_{A S}\right)^{\alpha_{1}^{A S}}+c_{2}^{A S}\left(A c l_{A S}\right)^{\alpha_{2}^{A S}} \\
O C_{A S} & =\left(c_{3}^{A S} F_{i n}+c_{4}^{A S}+c_{5}^{A S} F_{i n}+c_{6}\right)+c_{O 2}^{A S} R o_{A S}+c_{d}^{O R G} P_{X_{T}, V S S}
\end{aligned}
$$

Trickling filter The capital cost is a function of filter area $A_{T F}$ and clarifier are $A c l_{T F}$. Similar to the activated sludge, the operating cost has three terms: maintenance cost as a function of throughput, oxygen consumption, 
and sludge disposal cost.

$$
\begin{aligned}
I C_{T F} & =2\left(c_{1}^{T F} A_{T F}^{2}+c_{2}^{T F} A_{T F}+c_{3}^{T F}\right)+c_{4}^{T F} A c l_{T F}^{\alpha_{1}^{T F}} \\
O C_{T F} & =\left(c_{5}^{T F} F_{\text {in }}+c_{6}^{T F}\right)+c_{O 2}^{T F} R_{T F}\left(C_{\text {in }}-C_{\text {out }}\right) F_{\text {in }}+c_{d}^{O R G}\left(C_{\text {in }}-C_{\text {out }}\right) F_{\text {in }}
\end{aligned}
$$

As can be seen from these equations, the correlations (22)-(27) include bilinear and concave terms that are nonlinear and nonconvex. The cost model gains in accuracy despite the computational complexities compared to the simple equations in (1). To see this difference more clearly, we can compare the performance of activated sludge and trickling filter for the removal of organic contaminants shown in Figure 9. The investment cost as a function of inlet concentration for activated sludge under fixed operating parameters is shown in Figure 9a. It is clear that there is a significant increase in investment cost $(9.89 \%)$ over the range of concentration considered. Figure $9 \mathrm{~b}$ shows that trickling filter removal efficiency is also a function of inlet concentration when the recycle ratio is fixed to 0.2. Finally, Figure 9c we compare the operating costs of activated sludge and trickling filter as functions of flowrate only. The figure shows that there exists a crossover point between the two curves, which indicates that it is incorrect to estimate the operating cost as a linear function of the flowrate. As a result, the simplified model, which is only dependent on the flowrate across the treatment unit, may lead to suboptimal, and possibly to solutions with the incorrect selection of treatment technology.

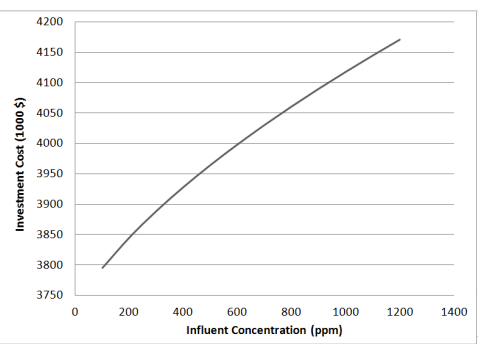

(a)

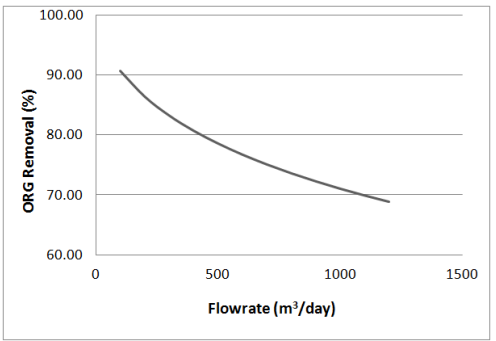

(b)

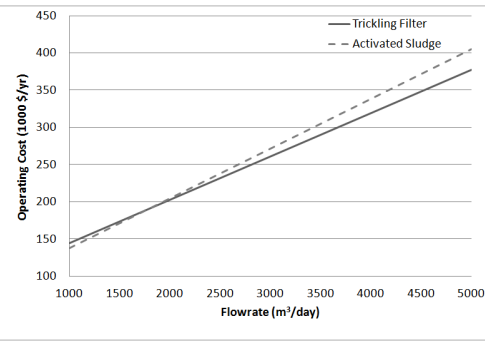

(c)

Figure 9: Organic treatment unit shortcut model comparison

\section{Computational strategies}

\subsection{Strategy for global optimal solution}

The resulting multi-scenario GDP problem (GDP-s) is given by the model in (GDP-1), where $h_{n}(\bullet)$ and $g_{n}(\bullet)$ are replaced by the short-cut equations in (2)-(21), and $f_{1}(\bullet)$ and $f_{2}(\bullet)$ are defined by the cost equations (22)-(27). Due to the presence of nonconvexities, sub-optimal solutions may be obtained if local solvers are used. Finding efficiently 
the global optimal solution in the proposed multi-scenario model would allow to solve the more accurate short-cut formulation.

The multi-scenario model (GDP-s) gives rise to a block diagonal structure, in which the design variables $\left(d_{r t}\right)$ are complicating variables in that they need to be accounted for in all scenarios. By defining the copy variables $d_{r t}^{m}, \hat{F}_{i}^{n}$, $y_{i}^{n}$, for each scenario, the problem can be reformulated as one with complicating constraints as shown in equation (28).

$$
\begin{aligned}
& d_{r t}^{n}=d_{r t}^{n+1} \quad \forall t \in T U, r=1, \ldots, R T_{t}, \forall n \in N, n<|N| \\
& \hat{F}_{i}^{n}=\hat{F}_{i}^{n+1} \quad \forall i, \forall n \in N, n<|N| \\
& y_{i}^{n}=y_{i}^{n+1} \quad \forall i, \forall n \in N, n<|N|
\end{aligned}
$$

This allows the application of the Lagrangean decomposition algorithm [28].

The proposed algorithm shown in Figure 10 involves an outer problem and an inner problem. The outer problem determines a global lower bound from a special relaxation problem $(R P)$ and fixes the selection of treatment unit options. The inner problem is then constructed for a fixed set of treatment unit technologies. The inner loop is solved with the Lagrangean decomposition algorithm and iterates between a lower bounding problem $\left(S P_{1}\right)-\left(S P_{N}\right)$ and an upper bounding problem $\left(P^{\prime \prime}\right)$. Since the predicted lower bounds are rigorous but exhibit dual gaps in the inner problem, global optimality can only be guaranteed within the predicted global upper and lower bounds after a maximum number of iterations.

\subsection{Subproblem descriptions}

Problem $(P)$ is a nonconvex MINLP that results from applying the hull reformulation[29] to (GDP-s). The MINLP relaxation problem $(R P)$ is obtained by replacing all the nonconvex terms present in $(P)$ with linear or convex underestimators so as to yield a valid lower bound. See Appendix for a summary of relevant nonconvex terms and their convex estimators. Problem $\left(S P_{n}\right)$ are MINLPs obtained by decomposing $(P)$ into $|N|$ scenarios using Lagrangean decomposition to obtain subproblems $\left(S P_{1}\right)-\left(S P_{|N|}\right)$. The complicating design variables $d_{r t}, F_{i}^{n}, I C_{t}^{T U}$ are replaced by their copy variables $d_{r t}^{n}, \hat{F}_{i}^{n}, I C_{t n}^{T U}$, respectively, as in constraints (28). The linking constraints (28) are dualized 


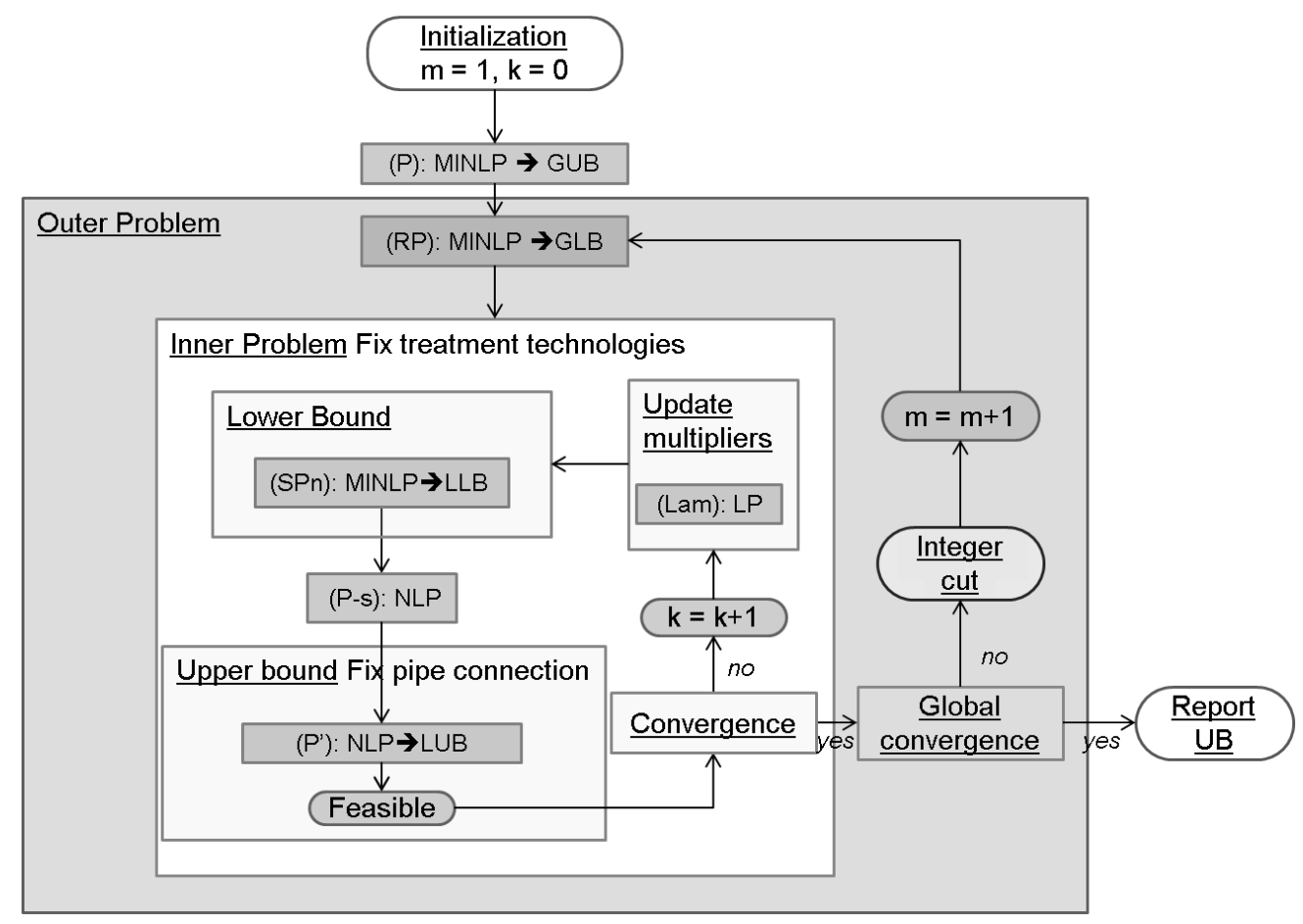

Figure 10: Decomposition scheme

and transferred to the objective function as shown in equation (29).

$$
\begin{array}{rl}
\operatorname{min.} z_{n}=p_{n} & A R\left[\sum_{t \in T U} I C_{t n}^{T U}+\sum_{i \in \text { Pipe }}\left(C_{i}^{\text {Pipe }} y_{i}^{n}+I C_{i}^{\text {Pipe }}\left(\hat{F}_{i}^{n}\right)^{\delta}\right)\right] \\
& +H p_{n} O C^{F W} F W_{n}+H p_{n} \sum_{i \in \text { Pipe }} O C^{\text {Pipe }} F_{i n}+p_{n} O C_{t n}^{T U} \\
& +\sum_{i}\left(\lambda_{i n}^{f}-\lambda_{i(n-1)}^{f}\right) \hat{F}_{i}^{n}+\sum_{i}\left(\lambda_{i n}^{y}-\lambda_{i(n-1)}^{y}\right) y_{i}^{n} \\
& +\sum_{i}\left(\lambda_{r t n}^{d}-\lambda_{r t(n-1)}^{d}\right) d_{r t}^{n}+\sum_{t \in T U}\left(\lambda_{t n}^{I C}-\lambda_{t(n-1)}^{I C}\right) I C_{t n}^{T U} \\
n=1, \ldots,|N|
\end{array}
$$

Problem $(P-s)$ can help to improve the solution quality of the upper bounding problem $\left(P^{\prime}\right) .(P-s)$ is an NLP that represents the simplified multi-scenario model. It applies effective contaminant removal ratios $\beta_{t j}^{n}$ and flow recovery ratios $R r_{t}^{n}$ from the subproblems $\left(S P_{n}\right)$, where $F_{\text {out }}=R r_{t} F_{i n}$. Problem $\left(P^{\prime}\right)$ is the NLP upper bounding problem in the inner iteration resulted from fixing all the integer variables in the original problem $(P)$. In problem ( Lam) the Lagrangean multipliers are updated using a hybrid algorithm based on the combination of cutting-plane and subgradient strategies described in Oliveira et al[30]. 


\subsection{Algorithm}

The steps of the proposed algorithm are as follows:

0. Initialization Determine bounds on variables $d_{r t}$ and $\hat{F}_{i}$ based on the numerical data provided in each water network. Set all multipliers $\lambda_{i n}^{f}, \lambda_{i n}^{y}$, and $\lambda_{r t n}^{d}$ to zero. Set outer iteration count $m=1$, inner iteration count $k=0$.

1. Global Upper Bound Fix all binary variables $y_{i}$ to 1 , and solve the MINLP problem $(P)$ in terms of the binary variables $Y_{r t}$ for the treatment units using non-global MINLP solvers such as DICOPT or a global solver such as BARON and LINDOGlobal with a large optimality gap (e.g. 70\%). If the time limit is exceeded, fix the binary variable $Y_{r t}$ to 1 for a subset of treatment technologies and solve problem $(P)$ again.

2. Global Lower Bound Solve the MINLP problem $(R P)$ to determine the global lower bound. Once the solution is obtained, fix the binary variables, $Y_{r t}$, for the inner problem.

3. Inner Problem Set $k=1$.

i. Solve the MINLP $\left(S P_{n}\right)$ for each scenario $n \in N$ to global optimality for the fixed treatment selection.

Three potential situations could result from this step. First, if all the subproblems are feasible and are solved to $\varepsilon_{1}$-tolerance within time limit, then we obtain for the selected treatment units a lower bound solution $Z^{L L B}$ by taking the sum of the subproblems' objective values $z_{n}^{*}$. If the problem is not solved within $\varepsilon_{1}$-tolerance in the specified time limit, then we convexify the subproblem as in problem $(R P)$ to form $\left(r S P_{n}\right)$, which can then be solved using a non-global MINLP solver. The third situation arises when any of the sub-problems is found to be infeasible, in which case the set of treatment selection is eliminated.

ii.(optional) Solve $(P-s)$ to local optimality.

iii. The binary variables $y_{i}$ in subproblem $\left(P^{\prime}\right)$ are fixed to zero if the flowrate capacity, $\hat{F}_{i}$, for a given pipe $i$ takes a value of zero in $(P-s)$. Solve the upper bounding problem $\left(P^{\prime}\right)$ to local optimality. Update $Z^{L U B}$.

iv. Check for convergence of the inner problem. If $Z^{L U B}<Z^{G U B}$, then update $Z^{G U B}=Z^{L U B}$. If $\left(Z^{L U B}-\right.$ $\left.Z^{L L B}\right) / Z^{L U B}<\varepsilon_{2}$ or if $Z^{L L B}>Z^{G U B}$, end inner loop.

v. Update the Lagrangean multipliers in (Lam). $k=k+1$ as described in [30].

4. Convergence Check global convergence criteria $\left(Z^{G U B}-Z^{G L B}\right) / Z^{G U B}<\varepsilon$, if the algorithm does not meet the $\varepsilon$-convergence criterion, add an integer cut $(30)$ to $(R P)$ to eliminates the current set of $Y_{r t}$. Reset all multipliers 


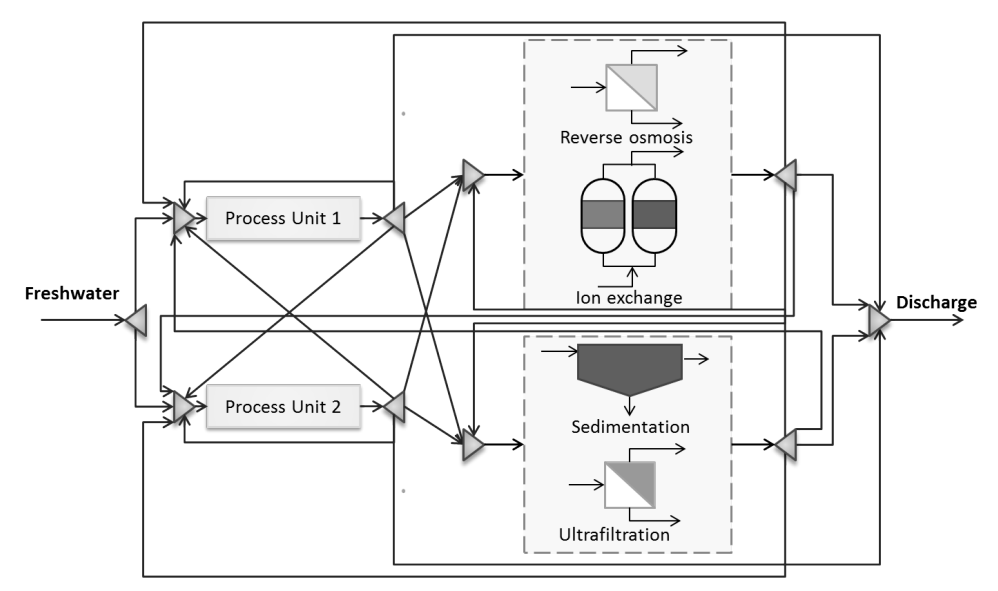

Figure 11: Example 1 network superstructure

to $0 . m=m+1$.

$$
\begin{aligned}
& \sum_{(r, t) \in B^{m}}\left(1-Y_{r t}\right)+\sum_{(r, t) \in N^{m}} Y_{r t} \geq 1 \quad \forall m=1, \ldots, M \\
& B_{r t}^{m}=\left\{(r, t) \mid Y_{r t}^{m}=1\right\} \quad N_{r t}^{m}=\left\{(r, t) \mid Y_{r t}^{m}=0\right\}
\end{aligned}
$$

\section{Numerical examples}

Three water network examples are provided to demonstrate the formulation and decomposition algorithm. Problem (GDP-1) was automatically reformulated as an MINLP using GAMS/EMP (Extended Mathematical Programming)[31], which is a modeling framework for automated mathematical reformulation. The MINLP models are formulated using GAMS 24.0[32] and solved on an Intel Core i7 2.93 GHz machine with 4.00 GB memory. CPLEX was used for the MILP problems, and DICOPT[33], LINDOGlobal[34] and BARON 11.1[21] are used to solve the MINLP problems. Note that while DICOPT is a non-global solver, LINDOGlobal and BARON are global optimization solvers.

\subsection{Example 1: illustrative example with short-cut treatment unit models}

In this example, we consider the problem structure presented in the illustrative example in Section 3. The process unit data are the same, but we now incorporate short-cut models of treatment units for TSS and TDS removal. TU1 involves as choices reverse osmosis and ion exchange for the removal of TDS, and TU2 involves sedimentation and ultrafiltration for the removal of TSS. The superstructure is shown in Figure 11.

The full problem and the decomposition algorithm subproblems' model statistics are shown in Table 10. The full MINLP, which is obtained with the hull reformation (GDP-s)[35] problem is solved using BARON to arrive at 
the solution of $\$ 434,164.59$ in 2,132 CPUs with a 5\% optimality gap. Then the problem is solved using the decomposition algorithm to global optimality. The first step is to obtain a god initial solution from problem $(P)$. This is accomplished by using LINDOGlobal terminating the search as soon as a feasible solution is found. The global upper bound $\$ 462,712.88$ is obtained in 93 CPUs. The convex MILP relaxation $(R P)$ provided a global lower bound of $\$ 352,346.66$. Ion exchange is chosen to remove TDS and ultrafiltration to remove TSS. For these choices the MINLP subproblems $\left(S P_{n}\right)$ is each solved with BARON with a 5\% optimality tolerance. They each yield an objective value of $\$ 146,711.84, \$ 142,964.20$, and $\$ 142,619.09$. Summing the values from the three scenarios yields a lower bound of $\$ 432,295,12$. We then solve the NLP optional step $(P-s)$ to reach a solution of $\$ 381,083.40$, a lower bound for this configuration. The objective value of this problem is not crucial to the algorithm since the treatment units are simplified, instead, we use this step to obtain stream connectivities for the NLP problem $\left(P^{\prime}\right)$ with that fixed configuration to obtain an upper bound. The NLP problem $\left(P^{\prime}\right)$ yields a solution of $\$ 433,173.72$ (shown in Figure 12a), which is a new upper bound. Since the lower bound $(\$ 352,346.66)$ lies below this upper bound, we add an integer cut to the convex MILP relaxation $(R P)$. Since this problem is infeasible within the updated global upper bound, the search is terminated in a total of 471 CPUs. Thus, the decomposition algorithm is able to reduce the computational effort by almost a factor of five (471 CPUs vs. 2,132 CPUs).

Table 10: Example 1: Subproblem model statistics

\begin{tabular}{lccccccc}
\hline \hline Subproblem & Formulation Type & UB/LB & Solution Type & \# Binary Vars & \# Cont Vars & \# Constraints & \# Non-convex Terms \\
\hline$(P)$ & MINLP & UB & Global & 24 & 465 & 610 & 198 \\
$\left(S P_{n}\right)$ & MINLP & LB & Global & 20 & 203 & 206 & 86 \\
$(P-s)$ & NLP & - & Local & - & 373 & 547 & 144 \\
$\left(P^{\prime \prime}\right)$ & NLP & UB & Local & - & 435 & 430 & 198 \\
\hline
\end{tabular}

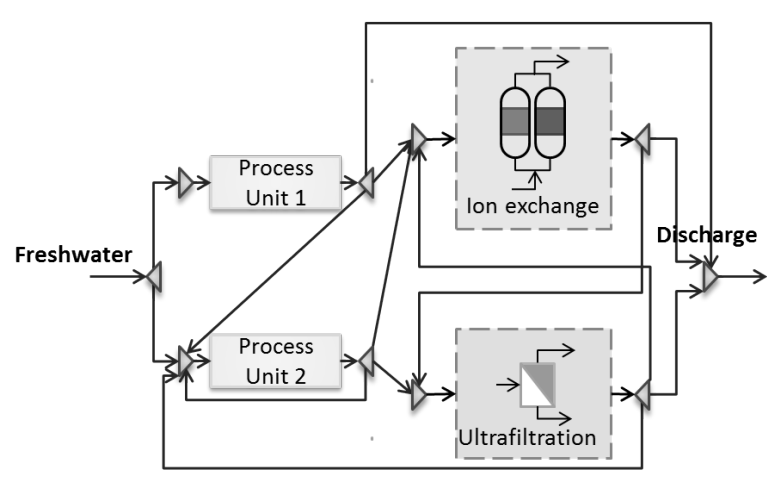

(a)

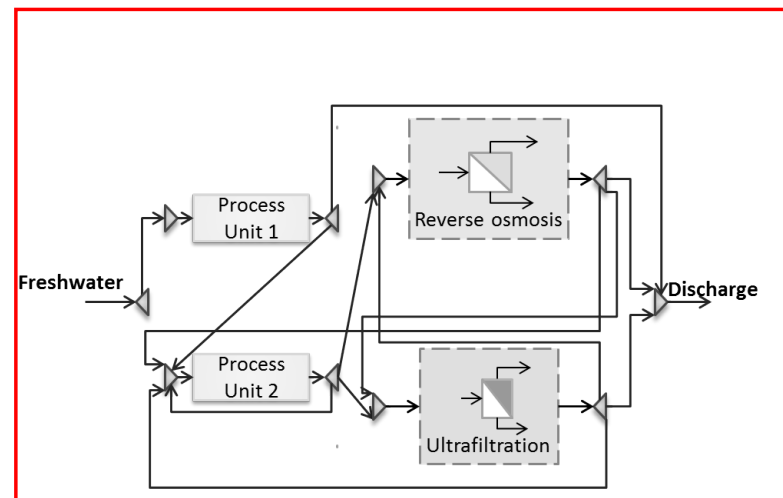

(b)

Figure 12: Example 1:(a) Short-cut model optimal solution (b) Simplified model optimal solution

In order to demonstrate the advantage of using short-cut models, we can compare its optimal solution with the result from the simplified model. To make the comparison on the same basis, the first step is to optimize the WN with 
simplified model, then in the second step, the WN with short-cut model is optimized with fixed network structure from step one. The optimized WN structure obtained using simplified models is shown in Figure 12b. As can be seen, the main difference is that the treatment unit chosen for TDS removal is reverse osmosis for the simplified model WN and ion exchange for the short-cut model WN. There are also two piping connections that are different in the result, namely, from PU1 to TU1 and from TU1 to PU2. The cost of the network increased by $2.5 \%$, from $\$ 430,157$ for configuration in Figure 12a to $\$ 440,884$ for configuration in Figure 12b. Note that the problem is optimized to within $1 \%$ optimality in order to ensure the validity of the comparison. Hence the short-cut WN has a cost of $\$ 430,157$ instead of $\$ 433,174$ as presented in the detailed decomposition algorithm steps.

Furthermore, the recoveries have a direct impact on treatment cost in practice. Thus, the purpose of using short-cut models is to calculate the wastewater treatment recoveries through optimization, whereas simplified models assume that they are fixed. Both specified recoveries in the simplified models and the calculated recoveries in the short-cut models are presented in Table 11. Column S1 represent the removal ratio specified in the simplified models; S2 shows the calculated recoveries from optimizing short-cut models with the fixed configuration in Figure 12b; and S3 represents the recoveries from directly optimizing short-cut models. From the table, we can see that the removal ratio upper bounds are reached (90\% for IX and $99 \%$ for UF) or nearly reached (99\% for RO) for the selected units due to

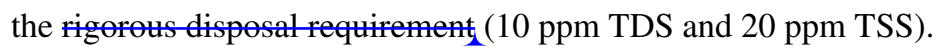

Table 11: Example 1: Recovery comparison

\begin{tabular}{lc|ccc|ccc|ccc}
\hline \hline Treatment Unit & \multicolumn{3}{|c|}{$\mathrm{n} 1$} & \multicolumn{3}{c|}{$\mathrm{n} 2$} & \multicolumn{3}{c}{ n3 } \\
\hline & & S1 & S2 & S3 & S1 & S2 & S3 & S1 & S2 & S3 \\
\hline \multirow{2}{*}{ TDS Removal } & RO & 80 & 98 & - & 90 & 98 & - & 99 & 98 & - \\
& IX & 70 & - & 90 & 80 & - & 90 & 90 & - & 90 \\
\multirow{5}{*}{ TSS Removal } & SE & 50 & - & - & 60 & - & - & 70 & - & - \\
& UF & 70 & 99 & 99 & 85 & 99 & 99 & 99 & 99 & 99 \\
\hline
\end{tabular}

\subsection{Example 2: metal finishing industry wastewater treatment}

The next example comes from a metal finishing industry located in Turkey[4]. Steel wheel production, tractor production, engine assembly shop, and spring production are the four main production lines that are involved. Each process results in a wastewater streams with various level of TSS, heavy metal (HM), TDS, and BOD. The worst, nominal, and best scenario concentration values are given in Table 12. This example considers only wastewater treatment network (no PU included) whose superstructure is shown in Figure 13. We apply the short-cut treatment models for the removal of TSS, TDS, and BOD. For HM removal we assume fixed recoveries. Note that TDS has the highest average concentration among the four groups of contaminant, thus it gives rise to the most difficult removal. On the contrary, 


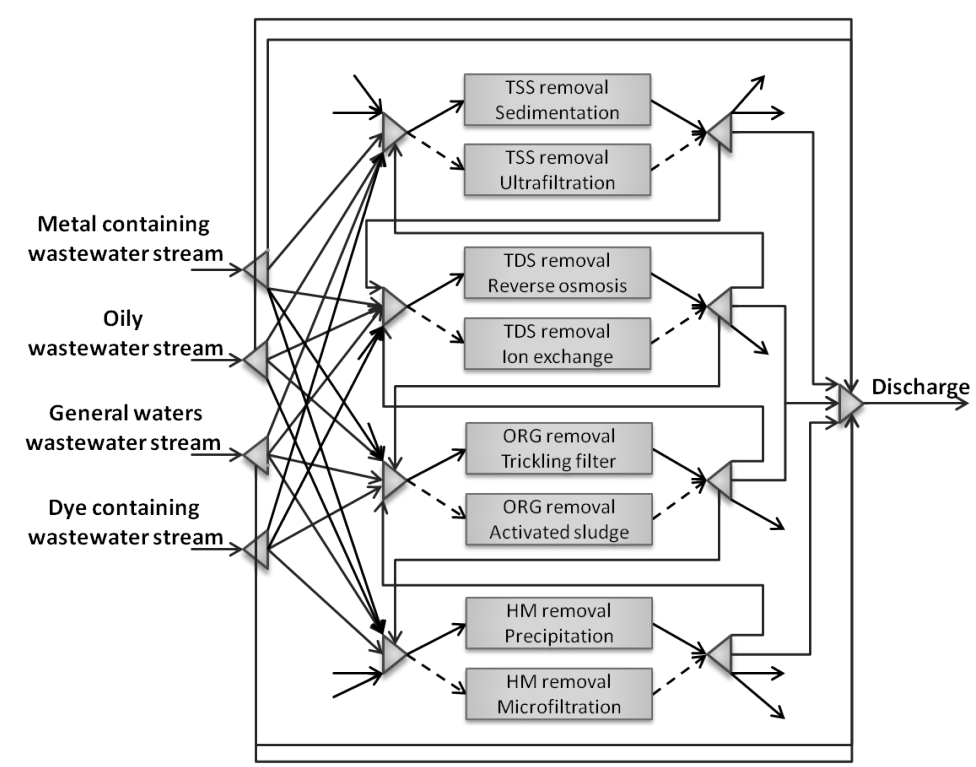

Figure 13: Example 2: Metal finishing wastewater treatment network superstructure

$\mathrm{HM}$ is the easiest contaminant to removal.

Table 12: Example 2: Metal finishing data

\begin{tabular}{|c|c|c|c|c|c|c|c|c|c|c|c|c|c|}
\hline \multirow[t]{3}{*}{ Stream } & \multirow[t]{3}{*}{ Flowrate (ton/h) } & \multicolumn{12}{|c|}{ Concentration (ppm) } \\
\hline & & \multicolumn{3}{|c|}{ TSS } & \multicolumn{3}{|c|}{ HM } & \multicolumn{3}{|c|}{ TDS } & \multicolumn{3}{|c|}{ BOD } \\
\hline & & $\mathrm{n} 1$ & n2 & n3 & $\mathrm{n} 1$ & $\mathrm{n} 2$ & n3 & $\mathrm{n} 1$ & $\mathrm{n} 2$ & n3 & $\mathrm{n} 1$ & $\mathrm{n} 2$ & n3 \\
\hline Metal Containing & 5.25 & 195 & 150 & 105 & 96.59 & 74.3 & 52.01 & 2405 & 1850 & 1295 & 78 & 60 & 42 \\
\hline Oily & 5.084 & 546 & 420 & 294 & 0 & 0 & 0 & 3250 & 2500 & 1750 & 260 & 200 & 140 \\
\hline General Waters & 3.96 & 39 & 30 & 21 & 14.3 & 11 & 7.7 & 1690 & 1300 & 910 & 58.5 & 45 & 31.5 \\
\hline Dye Containing & 3.3 & 136.5 & 105 & 73.5 & 6.5 & 5 & 3.5 & 6890 & 5300 & 3710 & 1950 & 1500 & 1050 \\
\hline Discharge Limit & & & 120 & & & 30 & & & 300 & & & 80 & \\
\hline
\end{tabular}

The corresponding MINLP using the hull reformulation[35] has 1,229 equations, 47 discrete variables, and 961 continuous variables. Solving $(P)$ directly does not yield a feasible solution using standard solvers such as DICOPT, KNITRO, or SBB. To facilitate computation, we fix all $y_{i}$ to 1 (i.e. all piping connections exist) and $Y_{r t}$ to 1 for ultrafiltration, reverse osmosis, activated sludge, and microfiltration. With these fixed values, we can obtain a global upper bound with an objective value of $\$ 304,405$ in 27.6 CPUs using LINDOGlobal with a $70 \%$ optimality tolerance. Problem $(R P)$ is solved subsequently and a global lower bound with an objective value of $\$ 98,790$ is obtained in 12.6 CPUs. The lower bound solution fixes ultrafiltration, ion exchange, activated sludge, and microfiltration as the technology selection for the inner iteration. In the operating range of this example, ion exchange has a higher capital cost and a worse removal performance. However, its operating cost is lower than that of the reverse osmosis. In addition, both technologies are capable of meeting the discharge limit. Thus, it is possible that the selections from the 


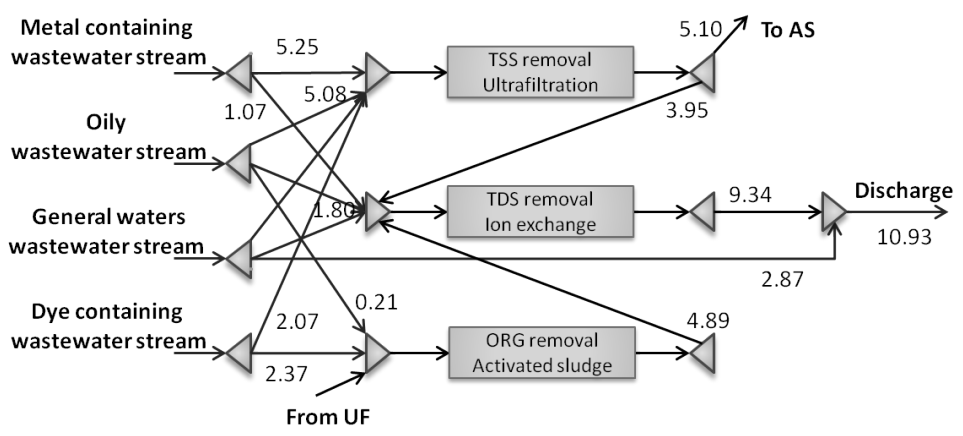

Figure 14: Example 2: Metal finishing water network configuration

lower bound outperforms the initial technologies chosen for $(P)$. For the new choices the MINLP subproblems $\left(S P_{n}\right)$ is each solved with BARON with a $10 \%$ optimality tolerance. They yield an objective value of $\$ 131,690, \$ 52,954$, and $\$ 46,226$. Summing the values from the three scenarios yields a lower bound of $\$ 230,870$. We then solve the NLP optional step $(P-s)$ to obtain the stream connectivities. Based on the configuration, the NLP problem $\left(P^{\prime}\right)$ yields an upper bound of $\$ 234,820$. With the integer cut to eliminate the current configuration, the subsequent outer problem $(R P)$ is found to be infeasible; thus, we have reached the global optimum. The entire algorithm requires 2,308.5 CPUs, where 1,608.0 CPUs is used to solve the lower bounding problem. In comparison, the original problem $(P)$ cannot be solved to optimality in the resource limit (7200 CPUs) with LINDOGlobal and BARON.

The resulting configuration is shown in Figure 14. Note that the recovery ratio in the ion exchange unit is chosen to be the lower bound 0.5 , which implies that it is cheaper to dispose rather than to treat the stream. Also, since the overall streams have HM concentration less than the discharge limit, no HM removal is required.

\subsection{Example 3: petroleum refinery water use}

We consider a modified refinery case study as the final example[6,36] and the units involved are shown in Figure 15. The primary water sources are freshwater and purified water. In addition, crude oil often carries emulsified water, and can be considered as a third process water source. Two sinks are considered for discharge, a centralized wastewater treatment facility on site and the nearby river. Five water-using process units are considered in this study. They are desalination, column condensation, steam generation, cooling water, and general consumption. The water quality and flowrate requirement of these processes are summarized in Table 13. The third water source, crude oil train, has a maximum flowrate of $15 \mathrm{ton} / \mathrm{h}$, and the wastewater treatment plant can accept a maximum of $360 \mathrm{ton} / \mathrm{h}$ of wastewater. Reverse osmosis and ion exchange remove salt from the streams, and trickling filter and activated sludge are used to remove organic content of the streams. 


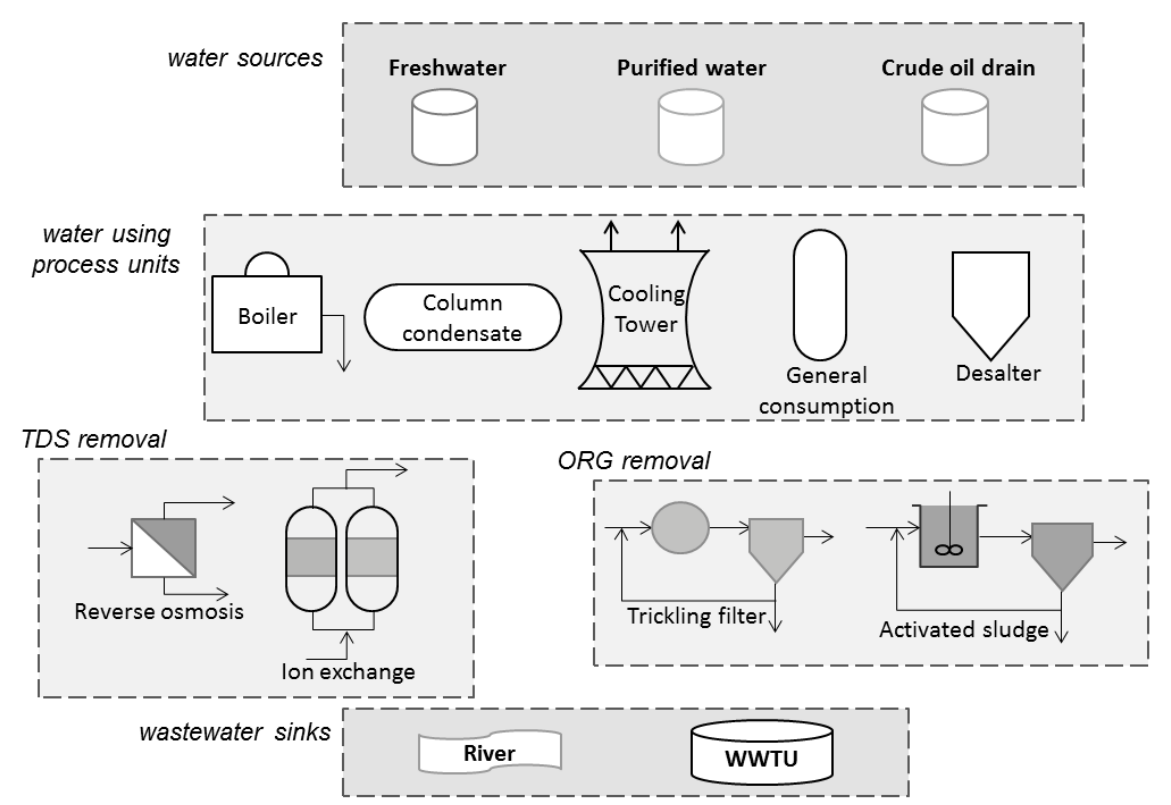

Figure 15: Example 3: Refinery process water system superstructure

Table 13: Example 3: Petroleum refinery data

(a) Process units

\begin{tabular}{|c|c|c|c|c|c|c|c|c|c|c|}
\hline & Flowrate (ton/h) & Loss (ton $/ \mathrm{h}$ ) & \multicolumn{6}{|c|}{ Discharge Load (kg/h) } & \multicolumn{2}{|c|}{$C_{i n}^{\max }(\mathrm{ppm})$} \\
\hline & & & & n1 & $n$ & & $\mathrm{n}$ & & & \\
\hline & & & TDS & ORG & TDS & ORG & TDS & ORG & TDS & ORG \\
\hline Boiler & 25 & 18 & 3.5 & 1.21 & 2.07 & 1.10 & 0.64 & 0.99 & 10 & 1 \\
\hline Condensate & 22.5 & 0 & 4.28 & 146.23 & 3.94 & 125.55 & 3.6 & 104.87 & 10 & 1 \\
\hline Cooling tower & 1000 & 405 & 615 & 219 & 310.9 & 110 & 6.8 & 1 & 2500 & 220 \\
\hline General consumption & 10 & 0 & 9.5 & 70 & 8.29 & 61.1 & 7.08 & 52.19 & 300 & 50 \\
\hline Desalter & 85 & 0 & 153 & 544 & 136.07 & 510.31 & 119.14 & 476.63 & 200 & 100 \\
\hline
\end{tabular}

(b) Concentration limit (ppm)

\begin{tabular}{l|rrr|rr}
\hline \hline & \multicolumn{3}{|c|}{ Sources } & \multicolumn{2}{c}{ Discharge sinks } \\
\hline & Fresh water & Purified water & Crude oil train & River & Wastewater treatment plant \\
TDS & 50 & 10 & 135 & 50 & 364 \\
ORG & 15 & 0 & 45 & 200 & 759 \\
\hline
\end{tabular}

The resulting MINLP has 1,768 equations, 1,331 continuous variables, and 85 binary variables. Note that the problem size is larger than that of Example 2. However, solving the problem directly using LINDOGlobal yields an optimal solution of $\$ 1,906,264$ with $5 \%$ optimality gap in 209 CPU s. The reason that the problem can be solved effectively without the decomposition algorithm can be seen in the resulting network configuration as shown in Figure 16. Trickling filter is chosen to remove ORG, and the highest removal ratio, $R_{c}^{O R G}=90 \%$, is selected. The consumption of freshwater is $360.5 \mathrm{t} / \mathrm{h}$, and the consumption of purified water is $47.5 \mathrm{t} / \mathrm{h}$, which is a $734.5 \mathrm{t} / \mathrm{h}$ reduction had reusing and recycling not been performed. First, the wastewater streams are reused and recycled within the network instead of 


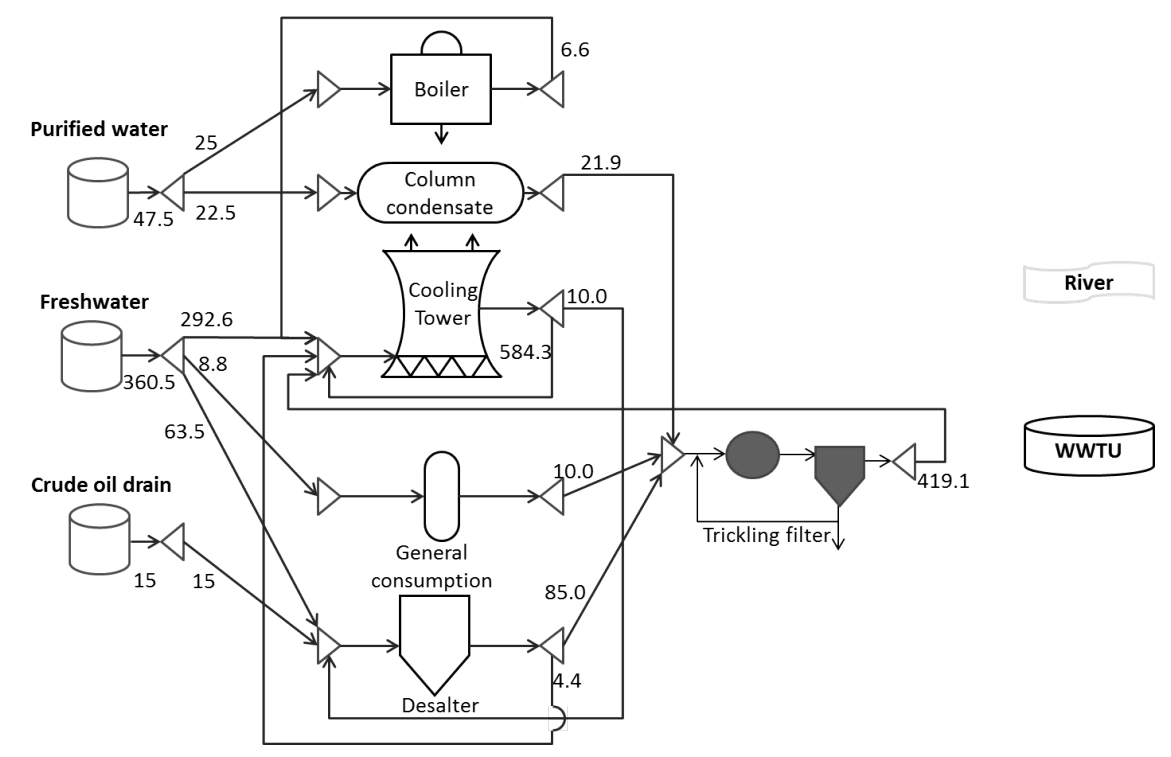

Figure 16: Example 3: Optimal network configuration for the refinery water system

being discharged to the wastewater sinks. Also, no salt-removal unit is selected in this configuration, which is due to the high TDS tolerance level at the inlet stream of the cooling tower, as well as the high rate of evaporation loss in the cooling tower. The cooling tower then reuses most of its outlet stream, leading to a cooling loop that is more efficient than a once-through design.

\section{Conclusion}

By considering the use of short-cut models for treatment units that remove TDS, TSS, and organics, we are able to exploit the trade-offs between treatment cost and removal efficiency of the units. The model (GDP-1) is developed to accommodate the modifications in the architecture and formulation of the treatment units. In order to solve the resulting formulation to global optimality, we have presented a Lagrangean-based decomposition algorithm that is tailored to the water network problem. Several examples are presented to demonstrate the effectiveness of the algorithm in improving the quality and computation effort of the solution.

\section{Nomenclature}

\section{Reverse Osmosis}

$\Delta P_{\text {drop }}$ Pressure drop along the membrane channel, $P a$ 
$\Delta \pi \quad$ Osmotic pressure difference across the membrane, $\mathrm{Pa}$

$N \quad$ Number of membranes

$P_{f} \quad$ Pressure at the feed side of the membrane, $P a$

$P_{p} \quad$ Pressure at the permeate side of the membrane, $\mathrm{Pa}$

\section{Ion Exchange}

A Resin cross-sectional area, $m^{2}$

$B V \quad$ Volume of water treated per volume of resin, Lwater/Lresin

$C T \quad$ Contact time, $h$

$M W_{a n}$ Molar mass of anion, $\mathrm{kg} / \mathrm{kmol}$

$M W_{c a}$ Molar mass of cation, $\mathrm{kg} / \mathrm{kmol}$

$q \quad$ Operating capacity of the resin, $e q /$ Lresin

SFR Service flow rate, $m^{3}$ water $/\left(m^{3}\right.$ resinh $)$

$S L R \quad$ Surface loading rate, $m / h$

$V \quad$ Resin volume, $m^{3}$

$V_{w w} \quad$ Volume of waste water treated

$X \quad$ Mass fraction in inlet water of ion wanted to be removed

\section{Ultrafiltration}

$\Delta P \quad$ Transmembrane pressure

$\mu \quad$ dynamic viscosity of water

$A_{m e m b}$ Membrane area

$k_{M} \quad$ membrane resistance coefficient

\section{Sedimentation}

A Volume of the filter media, $m^{2}$ 
$D \quad$ Depth, $m$

$N C \quad$ Volume of the filter media, $m^{2}$

OR Overflow rate, $m^{3} / m^{2} d a y$

$t \quad$ Nominal detention time, $h$

\section{Activated Sludge}

$\tau \quad$ Residence time, day

Acl Clarifier area, $m^{2}$

$P_{x, b i o} \quad$ Biomass wasted, $g /$ day

$P_{X_{T}, V S S}$ Total sludge wasted daily, $g /$ day

$R_{o} \quad$ Oxygen consumption, $g /$ day

SRT Solid retention time, day

$V \quad$ Aerator volume, $m^{3}$

$X \quad$ Biomass concentration in the aeration tank, $g / m 3$

$X_{T} \quad$ MLVSS concentration, $g / m 3$

$X_{0, i} \quad$ Influent nbVSS concentration, $g / m 3$

\section{Trickling Filter}

A Area of the filter media, $m^{2}$

Acl Clarifier area, $m^{2}$

$D \quad$ Depth, $m$

$E_{1} \quad$ Fraction of BOD removal for first stage, $\%$

$E_{2} \quad$ Fraction of BOD removal for second stage, $\%$

F Recirculation factor

$L_{B} \quad$ BOD loading to filter, $\mathrm{kg} B O D / \mathrm{m}^{3} \mathrm{~d}$ 
OR Overflow rate, $m / h r$

$R_{o} \quad$ Oxygen consumption, $\mathrm{kg} \mathrm{O}_{2} / \mathrm{kg}$ BOD applied

$R_{r} c y \quad$ Recirculation ratio

$V \quad$ volume of filter media, $m^{3}$

$W_{1} \quad$ BOD loading applied to the first-stage filter, $\mathrm{kg} / \mathrm{d}$

$W_{2} \quad$ BOD loading applied to the second-stage filter, $\mathrm{kg} / \mathrm{d}$

\section{Author Information}

Corresponding Author

*E-mail: grossmann@cmu.edu.

\section{Acknowledgments}

The authors would like to acknowledge financial support from the National Science Foundation for financial support under grant CBET $96524_{2}$ the program "Estancias de movilidad en el extranjero "Jose Castillejo" para jvenes doctores" (JC2011-0051) of the Spanish Ministerio de Educacin, and from the University of Alicante (GRE11-19).

\section{Appendix: Convex envelopes}

- Bilinear terms [37]: $F C \rightarrow f$

$$
\left.\begin{array}{l}
f \geq F^{\text {min }} C+C^{\text {min }} F-F^{\text {min }} C^{\text {min }} \\
f \geq F^{\text {max }} C+C^{\text {max }} F-F^{\text {max }} C^{\text {max }} \\
f \leq F^{\text {min }} C+C^{\text {max }} F-F^{\text {min }} C^{\max } \\
f \leq F^{\text {max }} C+C^{\text {min }} F-F^{\text {max }} C^{\text {min }}
\end{array}\right\}
$$

- Concave term: $F^{\alpha} \rightarrow \Theta$

$$
\Theta \geq\left(F^{\min }\right)^{\alpha}+\left(\frac{\left(F^{\max }\right)^{\alpha}-\left(F^{\min }\right)^{\alpha}}{F^{\max }-F^{\min }}\right)\left(F-F^{\text {min }}\right)
$$


- $\sqrt{V} \rightarrow V_{\text {new }}$ (From trickling filter formulation (17) and (19))

$$
V_{\text {new }}^{2}-V \leq 0
$$

\section{References}

[1] ERM CDP Water Disclosure 2010 Global Report.; tech. rep.; Carbon Disclosure Projects, 2010.

[2] Karuppiah, R.; Grossmann, I. E. Global optimization for the synthesis of integrated water systems in chemical processes. Computers \& Chemical Engineering 2006, 30, 650 -673.

[3] Alva-Argez, A.; Vallianatos, A.; Kokossis, A. A multi-contaminant transhipment model for mass exchange networks and wastewater minimisation problems. Computers \& Chemical Engineering 1999, 23, 1439 -1453.

[4] Galán, B.; Grossmann, I. E. Optimal Design of Distributed Wastewater Treatment Networks. Industrial \& Engineering Chemistry Research 1998, 37, 4036-4048.

[5] Takama, N.; Kuriyama, T.; Shiroko, K.; Umeda, T. Optimal water allocation in a petroleum refinery. Computers \& Chemical Engineering 1980, 4, $251-258$.

[6] Huang, C.-H.; Chang, C.-T.; Ling, H.-C.; Chang, C.-C. A Mathematical Programming Model for Water Usage and Treatment Network Design. Industrial \& Engineering Chemistry Research 1999, 38, 2666-2679.

[7] Savelski, M.; Bagajewicz, M. On the necessary conditions of optimality of water utilization systems in process plants with multiple contaminants. Chemical Engineering Science 2003, 58, 5349 -5362.

[8] Faria, D. C.; Bagajewicz, M. J. Planning Model for the Design and/or Retrofit of Industrial Water Systems. Industrial \& Engineering Chemistry Research 2011, 50, 3788-3797.

[9] Khor, C. S.; Shah, N.; Mahadzir, S.; Elkamel, A. Optimisation of petroleum refinery water network systems retrofit incorporating reuse, regeneration and recycle strategies. The Canadian Journal of Chemical Engineering 2012, 90, 137-143.

[10] Dzhygyrey, I.; Jezowski, J.; Kvitka, O.; Statyukha, G. In 19th European Symposium on Computer Aided Process Engineering; Jezowski, J., Thullie, J., Eds.; Computer Aided Chemical Engineering, Vol. 26; Elsevier, 2009, pp $853-858$.

[11] Jezowski, J. Review of Water Network Design Methods with Literature Annotations. Industrial \& Engineering Chemistry Research 2010, 49, 4475-4516. 
[12] Bagajewicz, M. A review of recent design procedures for water networks in refineries and process plants. Computers \& Chemical Engineering 2000, 24, 2093 -2113.

[13] Foo, D. C. Y. State-of-the-Art Review of Pinch Analysis Techniques for Water Network Synthesis. Industrial \& Engineering Chemistry Research 2009, 48, 5125-5159.

[14] Davis, M. L. Water and Wastewater Engineering, Design Princicples and Practices; McGraw-Hill, 2010.

[15] Saif, Y.; Elkamel, A.; Pritzker, M. Optimal design of reverse-osmosis networks for wastewater treatment. Chemical Engineering and Processing: Process Intensification 2008, 47, 2163 -2174.

[16] Karuppiah, R.; Grossmann, I. E. Global optimization of multiscenario mixed integer nonlinear programming models arising in the synthesis of integrated water networks under uncertainty. Computers \& Chemical Engineering 2008, 32, $145-160$.

[17] Faria, D. C.; Bagajewicz, M. J. On the appropriate modeling of process plant water systems. AIChE Journal 2010, 56, 668-689.

[18] Integrated Pollution Prevention and Control Reference Document on Available Techniques in Common Waste Water and Waste Gas Treatment/Management Systems in the Chemical Sector.; tech. rep.; European Commission, 2011.

[19] Faria, D. C.; Bagajewicz, M. J. A new approach for global optimization of a class of MINLP problems with applications to water management and pooling problems. AIChE Journal 2012, 58, 2320-2335.

[20] Ahmetović, E.; Grossmann, I. E. Global superstructure optimization for the design of integrated process water networks. AIChE Journal 2011, 57, 434-457.

[21] Sahinidis, N. BARON: A general purpose global optimization software package. Journal of Global Optimization 1996, 8, 201205.

[22] Vince, F.; Marechal, F.; Aoustin, E.; Brant, P. Multi-objective optimization of RO desalination plants. Desalination 2008, 222, $96-118$.

[23] John C. Crittenden, D. W. H. K. J. H., R. Rhodes Trussell; Tchobanoglous, G. MWHs Water Treatment Principles and Design; Wiley, 2012.

[24] George Tchobanoglous, F. L. B.; Stensel, H. D. Wastewater Engineering: Treatment and Reuse; McGraw-Hill, 2003.

[25] Qasim, S. R. Wastewater Treatment Plants: Planning, Design, and Operation, Second; CRC Press, 1998. 
[26] Sharma, J. R. Development of a Preliminary Cost Estimation Method for Water Treatment Plants. MA thesis, The University of Texas at Arlington, 2010.

[27] SuperPro Designer Users Guide.; Intelligen, Inc, 2007.

[28] Terrazas-Moreno, S.; Trotter, P. A.; Grossmann, I. E. Temporal and spatial Lagrangean decompositions in multisite, multi-period production planning problems with sequence-dependent changeovers. Computers \& Chemical Engineering 2011, 35, 2913 -2928.

[29] Grossmann, I. E.; Ruiz, J. P. In Mixed Integer Nonlinear Programming; Lee, J., Leyffer, S., Eds.; The IMA Volumes in Mathematics and its Applications, Vol. 154; Springer New York, 2012, pp 93-115.

[30] Oliveira, F.; Gupta, V.; Hamacher, S.; Grossmann, I. A Lagrangean decomposition approach for oil supply chain investment planning under uncertainty with risk considerations. Computers \& Chemical Engineering 2013, 50, $184-195$.

[31] EMP, Users Manual.; GAMS Development Corporation (2009), 2009.

[32] Brooke, A.; Kendrick, D.; Meeraus, A.; Raman, R. GAMS: A Users Guide.; GAMS Development Corporation, 2012.

[33] Viswanathan, J.; Grossmann, I. A combined penalty function and outer-approximation method for MINLP optimization. Computers \& Chemical Engineering 1990, 14, 769 -782.

[34] LINDO Driver.; Lindo Systems Inc, 2011.

[35] Grossmann, I. E.; Trespalacios, F. Systematic modeling of discrete-continuous optimization models through generalized disjunctive programming. AIChE Journal 2013, 59, 3276-3295.

[36] Ullmer, C.; Kunde, N.; Lassahn, A.; Gruhn, G.; Schulz, K. WADO ${ }^{\mathrm{TM}}$ : water design optimization methodology and software for the synthesis of process water systems. Journal of Cleaner Production 2005, 13, 485 -494.

[37] McCormick, G. P. Computability of global solutions to factorable nonconvex programs: Part I Convex underestimating problems. Mathematical Programming 1976, 10, 147-175. 